\title{
Spatiotemporal Structure of Somatosensory Responses of Many-Neuron Ensembles in the Rat Ventral Posterior Medial Nucleus of the Thalamus
}

\author{
Miguel A. L. Nicolelis and John K. Chapin \\ Department of Physiology and Biophysics, Hahnemann University, Philadelphia, Pennsylvania 19102-1192
}

Classically, the rat ventral posterior medial (VPM) nucleus of the thalamus has been considered as a simple passive relay for single-whisker information to the primary somatosensory cortex (SI). However, recent reports have suggested that the VPM could contain a much more coarsely coded and spatiotemporally complex representation of the rat whisker pad. To address this possibility properly, we have carried out chronic simultaneous recordings of large numbers (up to 23) of single neurons, distributed across the entire VPM, in both awake and lightly anesthetized adult rats. Quantitative, computer-based reconstruction of receptive fields (RFs) revealed that single VPM neurons exhibit significant responses to discrete stimulation of as many as 20 single whiskers (mean \pm SD RF size, $13.7 \pm 4.8$ whiskers). By defining multiple response magnitude (RM) thresholds it was possible to subdivide these large VPM RFs quantitatively into a prominent center (mean \pm SD, $1.41 \pm 0.70$ whiskers, RM $>95 \%$ ) and an excitatory surround (up to 18 whiskers, RM $<95 \%$ ). VPM neurons exhibited both shortlatency responses (SLRs, from 4 to $10 \mathrm{msec}$ poststimulus) and/or long-latency responses (LLRs, 15-25 msec), each followed by inhibitory responses. Though LLRs were weaker (mean \pm SD, $47.19 \pm 33.34 \mathrm{~Hz}$ ) than SLRs (119.63 \pm 50.12 $\mathrm{Hz}$ ), they often defined RFs that differed considerably from those defined by the SLRs of the same cell. In particular, VPM cells with short-latency RFs centered in caudal whiskers (e.g., C1, D1, E1) showed a poststimulus time-dependent shift of these RF centers toward the rostral whiskers (e.g., C4, D4, E4). These caudal-to-rostral $(C \rightarrow R)$ RF shifts occurred in neurons with the largest RFs of our sample (17.2 \pm 2.4 whiskers). On the other hand, VPM cells with shortlatency RFs centered in rostral whiskers had the smallest RFs (13.1 \pm 4.1 whiskers) and usually did not exhibit timedependent RF center shifts. Multivariate analysis revealed that these two groups of VPM neurons, $C \rightarrow R$ shifting and rostral position (RP) cells, could be statistically distinguished according to a combination of three RF attributes (short-

Received Aug. 23, 1993; revised Dec. 6, 1993; accepted Dec. 16, 1993.

This work was supported by Grants NS-26722, AFSOR-90-0266, and AA06965 to J.K.C. We thank Dr. Rick C. S. Lin for his comments. We also acknowledge the continuous technological support provided by Dr. Donald Woodward and Dr. Samuel Deadwyler, and the technical assistance from Dr. Ann Kosobud, Terry Bunn, Dr. Robert Hampson, Dr. Steven Sawyer, Larry Andrews (NB Labs), IIarvey Wiggins (Spectrum Scientific), and Dr. Laura M. O. Oliveira.

Correspondence should be addressed to Miguel A. L. Nicolelis, M.D., Ph.D., Department of Physiology and Biophysics, Hahnemann University, MS 409, Broad and Vine Streets, Philadelphia, PA 19102-1192.

Copyright (C) 1994 Society for Neuroscience $0270-6474 / 94 / 143511-22 \$ 05.00 / 0$ latency RF center location, RF size, and magnitude of RF center shift). Quantitative, computer-based reconstruction of "population response maps" demonstrated that the "place" coding for each single whisker in the VPM involved a distinct weighted contribution from a large proportion of the simultaneously recorded neurons. Furthermore, singlewhisker stimulation induced a complex spatiotemporal wave of sensory-evoked activity that involved a much larger area of the VPM than a single barreloid, the classical anatomical representation of a single whisker in this nucleus. These results argue against the classical view that the rat VPM contains a highly topographic representation of single facial whiskers. Instead, they suggest that somatosensory information is processed in a much more distributed manner by ensembles of functionally heterogeneous populations of VPM neurons. Our hypothesis is that spatiotemporally complex multiwhisker RFs in the VPM emerge from asynchronous convergence of multiple ascending and descending afferents. As such, the functional properties of the VPM observed here may reflect dynamic processing of sensory information at multiple levels of the somatosensory network.

[Key words: neural networks, receptive fields, somatosensory system, plasticity, trigeminal system, thalamocortical relationships]

The rat ventral posterior medial (VPM) nucleus, the primary thalamic relay of the ascending trigeminal pathway, is a unique brain structure in that it contains a single class of projection (thalamocortical relay) cells (Harris, 1986), and is almost completely devoid of inhibitory interneurons (Harris, 1986; Benson et al., 1992). Projection neurons in the VPM are clustered in multiple cytochrome oxidase (CO)-rich cylinders (less than 200 $\mu \mathrm{m}$ in diameter) oriented rostrocaudally in the nucleus (Land and Simons, 1985). These cylinders, or "barreloids" (Van der Loos, 1976), resemble the CO-rich rods observed in the primate VPM (Rausell and Jones, 1991a,b). In the rat VPM, the barreloid distribution reproduces the spatial arrangement of facial whiskers in the animal's snout (Belford and Killackey, 1979a,b; Land and Simons, 1985). This discrete "anatomical map" of the face in the VPM is in somatotopographic register with similar maps located in the face representation region of the "barrel fields" in the primary somatosensory (SI) cortex (Woolsey and Van der I oos, 1970) and the "barrelets" in brainstem trigeminal subnuclei (Belford and Killackey, 1979a,b; Arvidson, 1982).

The distinct organization of CO-rich patches in the rat VPM, as well as in other levels of the trigeminal pathway, has led to the suggestion that low-threshold tactile information in this ro- 
dent is independently conveyed through multiple parallel sensory channels in the VPM to the SI cortex (Waite, 1973a; Rhoades et al., 1987; Killackey et àl., 1990; Chiaia et al., 1991b). In this scenario, sensory information from a single facial whisker is exclusively transmitted through its respective single barrelet $\rightarrow$ barreloid $\rightarrow$ barrel channel. As such, very little functional overlap is considered to occur between channels at any level of this lemniscal trigeminal pathway.

According to this assumption, the only region of the VPM excited by stimulation of a single facial whisker should be a single longitudinal CO-rich cylinder. Thus, receptive fields (RFs) in the VPM should be restricted to a single whisker, implying that the overall functional organization of this nucleus is based on "local" neuronal coding (Churchland and Sejnowski, 1992). Sensory systems based on this type of processing strategy rcly heavily on a high degree of single-cell specialization, defined by small and nonoverlapping RFs. Furthermore, neurons in the VPM should exhibit functionally homogeneous properties, in terms of the spatial and temporal characteristics of their RFs.

Recent neuroanatomical and neurophysiological findings, however, seem to argue against this classical model of organization of the rat VPM. Individual VPM cells have spherical or bitufted dendritic trees that extend for about $180 \mu \mathrm{m}$ from the cell soma (Chiaia et al., 1991b) and hence should be able to sample information from multiple CO-rich patches. Ascending information, including multiwhisker signals, from several subdivisions of the brainstem trigeminal complex has been demonstrated to converge upon VPM cells (Peschanski et al., 1983; Peschanski, 1984; Chiaia et al., 1991a), and to terminate in somewhat segregated dendritic territories (Williams et al., 1991). In addition, extensive fecdback projections from the primary somatosensory (SI) cortex, which by far outnumber the ascending trigeminothalamic projections, also converge on these VPM neurons (Chmielowska et al., 1989). Since this nucleus lacks interneurons (Benson et al., 1992), postexcitatory inhibition in the VPM is mainly derived from GABAergic neurons located in the reticular nucleus (Rt) of the thalamus (Minderhoud, 1971), which exhibit very complex multiwhisker RFs (Fisher et al., 1992).

Several groups have independently examined the properties of VPM neurons using electrophysiological approaches. Classical mapping studies reported that a vast majority of VPM cells exhibit single-whisker RFs (Waite, 1973a; Rhoades et al., 1987; Sugitani et al., 1990; Chiaia et al., 1991b), providing support for the local-coding model of thalamic processing. These results, however, were obtained in nonquantitative, unit/unit-cluster mapping recordings carried out without controlled stimulus delivery in anesthetized preparations. When similar studies were carried out using controlled, discrete stimulation of single whiskers and quantitative analysis of neuronal responses, a majority of VPM neurons were found to have multiwhisker RFs (Simons and Carvell, 1989; Armstrong-James and Callahan, 1991; Diamond et al., 1992; Nicolelis et al., 1993a). Such large RFs would be consistent with a "distributed" mode of sensory information coding.

Heretofore, the investigation of such an alternative model of information coding in the rat VPM has been hampered by the limitations of traditional neurophysiological recording techniques. Traditionally, single neurons are recorded serially, and their sensory properties are measured through repeated stimuli. Long-term stable single-unit recordings are difficult to obtain with this technique and rather strict and complex controls are necessary to ensure that nonstationary parameters, such as the level of anesthesia, do not influence the characterization of RFs. This limits the time available for RF characterization, reducing the cell yield per animal. More importantly, it precludes a proper reconstruction of the overall spatiotemporal representation of sensory stimuli embedded in ensembles of single VPM neurons.

To overcome the limitations of serial single-unit recording, we have developed techniques for large-scale parallel recordings that allow simultaneous sampling from up to 64 single neurons over long time periods in both awake and anesthetized animals (Nicolelis et al., 1993a-c). Here, this experimental approach was utilized to investigate whether the functional properties of ensembles of simultaneously recorded VPM neurons are strictly defined by the discrete arrangement of barreloids, as suggested by the classical local-coding model. To address this question in a quantitative way, a variety of new analytical methods were developed, including 3-D and 4-D graphical representations of single-cell and population RFs. In addition, multivariate statistical algorithms, such as factor and principal component analysis, were used to derive functional classifications of VPM cells based on multiple RF properties.

A preliminary report of dymamic properties of VPM RFs in awake rats was published elsewhere (Nicolelis et al., 1993a).

\section{Materials and Methods}

Electrodes. Chronic recordings were performed using probes (NBLABS, Dennison, TX) containing one or two bundles with eight $25-50 \mu \mathrm{m}$ Teflon-coated, stainless steel microwires per bundle. Each probe (Fig. $1 A$ ) was built by first soldering microwires to a 20 -pin plastic connector ( $15 \mathrm{~mm}$ long and $4 \mathrm{~mm}$ wide), providing up to 16 wires for extracellular neuronal recordings, one ground contact, and three pins for stimulating wires or EMG electrodes. After soldering, two bundles of eight microwires each were individualized with pieces of plastic tubing (4-5 $\mathrm{mm}$ in length). Polyethylene glycol was used to cover the contacts of the microwires with the connector and the initial segments of the bundles, including the plastic tubing.

Animals and surgical procedures. Adult Long-Evans (hooded) rats (250-300 gm) were used in all experiments. All animals received implants of bundles of microwires in the ventral posterior complex of the thalamus, during a short-duration surgery $(2-4 \mathrm{hr})$, targeting primarily the whisker representation area of the VPM nucleus. For this surgical procedure, animals were first anesthetized with a single intraperitoneal injection of pentobarbital $(50 \mathrm{mg} / \mathrm{kg}$ ) and then transferred to the stereotaxic apparatus. Usually, this dose was sufficient to maintain anesthesia throughout the surgical procedure. When necessary, deep anesthesia was maintained with small supplementary intraperitoneal injections $(0.1 \mathrm{ml})$ of pentobarbital. Following a single midsagittal skin incision, and retraction of soft tissue, the periosteum of the skull was removed. A small round craniotomy was then performed on the left parietal bone, $2.5-4.0 \mathrm{~mm}$ caudal to the bregma and $2.0-3.0 \mathrm{~mm}$ lateral to midline to allow the vertical implant of the microwire bundles in the VPM thalamus (Paxinos and Watson, 1986). Meanwhile, four to six stainless steel screws (Small Parts, Miami, FL) were firmly attached to the skull. They were used to ensure proper anchoring of the probe and also for ground purposes. Just prior to the implantation, the microwires were cut, using a new blade or sharp scissors, in a staggered arrangement so that wire length varied around an appropriate range (6.5-7.5 minn) to cover multiple cell clusters or "barreloids" in the thalamus (Fig. 2). To sample the VPM more thoroughly, the two bundles of eight microwires were also kept $\mathrm{l} \mathrm{mm}$ apart in the longitudinal axis of the probe. Subsequently, the microwires (except their tips) were soaked with a 20 $30 \%$ solution of sucrose, air dried, and mounted in the stereotaxic apparatus. Once dried, the sucrose helped to stiffen the bundles and facilitate the probe penetration through the dura. The bundles were then driven slowly (around $100 \mu \mathrm{m} / \mathrm{min}$ ) through the brain until the VPM was reached. A continuous monitoring of single- and multiunit recording was carried out throughout the surgery to evaluate the position of each microwire. Usually, good quality single and multiunits were recorded under these acute conditions allowing the final position of the microwires to be determined by the identification of facial RFs. Once the 
microwires had reached their target, the craniotomy was sealed with bone wax, and the probe was firmly attached to the skull using dental cement. At this point, two stainless steel, Teflon-coated wires $(0.005$ inch bare, 0.007 inch coated; A-M Systems, Inc., WA) were implanted in two different facial muscles surrounding the whisker pad of each animal (usually the $\mathrm{m}$. levator labii superioris and the $\mathrm{m}$. transversus nasi) (Dorfl, 1982) for EMG recordings. The skin was then loosely sealed around the probe and the animal was allowed to recover for 5-6 d before recording procedures started. This recovery period produces a considerable improvement in the quality of single units recorded with microwires.

Instrumentation for chronic recordings. Following the recovery period, animals were transferred to a recording chamber where all experiments were carried out. A head stage (NBLABS, Dennison, TX), containing 16 standard field effect transistors (FETs; Motorola MMBF5459), was used to connect the 20-channel plastic connector, cemented in the animal's head, to a preamplifier (gain 50 , bandpass $100 \mathrm{~Hz}$ to $8 \mathrm{kHz}$ ) located on the top of the recording chamber. The FETs were configured as current amplifying source followers with unity gain. They were connected to the preamplifier through high-fidelity, audio-connecting TVC insulated cables. Preamplifier outputs were connected, through ribbon cables, to differential AC inputs located in a Multi-channel Neuronal Acquisition Processor (MNAP) system (Spectrum Scientific, Dallas, TX), which was responsible for on-line multichannel neuronal spike sorting. The MNAP configuration provided inputs for up to 64 electrode wires. Two input boards provided programmable gain and additional bandpass filtering (typically set at $500 \mathrm{~Hz}$ to $5 \mathrm{kHz}$ ) for 32 signals each. A programmable switching network permitted routing of selected signals from the input boards to digital signal processing (DSP) channels for spike discrimination. To help reject movement artifacts caused by active behaving animals, programmable selection of wires with no spike activity for use as local references differenced to active wires was also supported by the switching network. The final configuration of the MNAP used here included two DSP boards, each with four Motorola DSP56001 microprocessors and eight A/D converters per DSP for a total of 64 spike discriminator channels. Signals were sampled simultaneously by all converters at a $40 \mathrm{kHz}$ rate. Control software for the MNAP box ran on a host 486 IBM PC-compatible microcomputer, allowing digital control of signal to channel assignment, signal gain, filtering, and window discrimination parameters. All spike discriminator programs were downloaded from the host to the DSP microprocessors.

Single-unit discrimination. Overall, the design of our chronic implants aimed at providing a wider sample area across the entire VPM (Fig. 2) by maximizing the simultaneous sample of single units recorded per animal. Elaborate time/voltage window discriminators (three voltage, one time; see Fig. $3 A$ ) were used to discriminate spike waveforms from single thalamic neurons. Given the filtering band used in these experiments $(500 \mathrm{~Hz}$ to $5 \mathrm{kHz})$, thalamic spike duration ranged from 0.8 to $1.5 \mathrm{msec}$. Strict precautions were taken to record only neuronal spikes and not axonal firing (Armstrong-James and Callahan, 1991). Since the microwires forming a bundle had different lengths, electrode tips were placed $100-500 \mu \mathrm{m}$ apart (as judged by histological reconstruction) in the VPM. This reduced the possibility of recording the same cell with different wires.

Time-voltage window discrimination was associated with an on-line principal component (PCA)-based spike-sorting algorithm (Abeles and Goldstein, 1977) for single-unit waveform identification (Nicolelis et al., 1993a). This technique allowed an on-line statistical characterization of each new waveform recorded per channel. It also allowed a continuous statistical follow-up of single-cell waveforms over long periods of time. To apply this algorithm, we first collected a sample of 200 waveforms derived from different cells located in different regions of the brain. The waveforms representing these spikes were then aligned on time (from 0 to $0.8 \mathrm{msec}$ ), and finally divided into 32 sequential $25 \mu \mathrm{sec}$ segments (used as variables by the PCA). From this database, three orthogonal principal components (each containing 32 coefficients) were derived using a standard PCA analysis (css:statisticA, Statsoft, Tulsa, OK) During chronic recordings, each new waveform, recorded on line from a given microwire, was simultaneously digitized into 32 points whose values were then multiplied by the corresponding 32 coefficients of each of the first three components previously calculated. Adding up all 32 value-coefficient products defined a principal component score for a particular waveform. The first two of these scores, for each waveform recorded per channel, was then plotted in real time, as an $x-y$ scatter plot. Usually, this method revealed the presence of multiple, statistically independent clusters of waveforms from the same microwire (Fig. $3 B$ ),

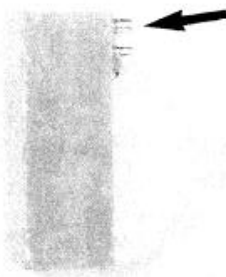

A
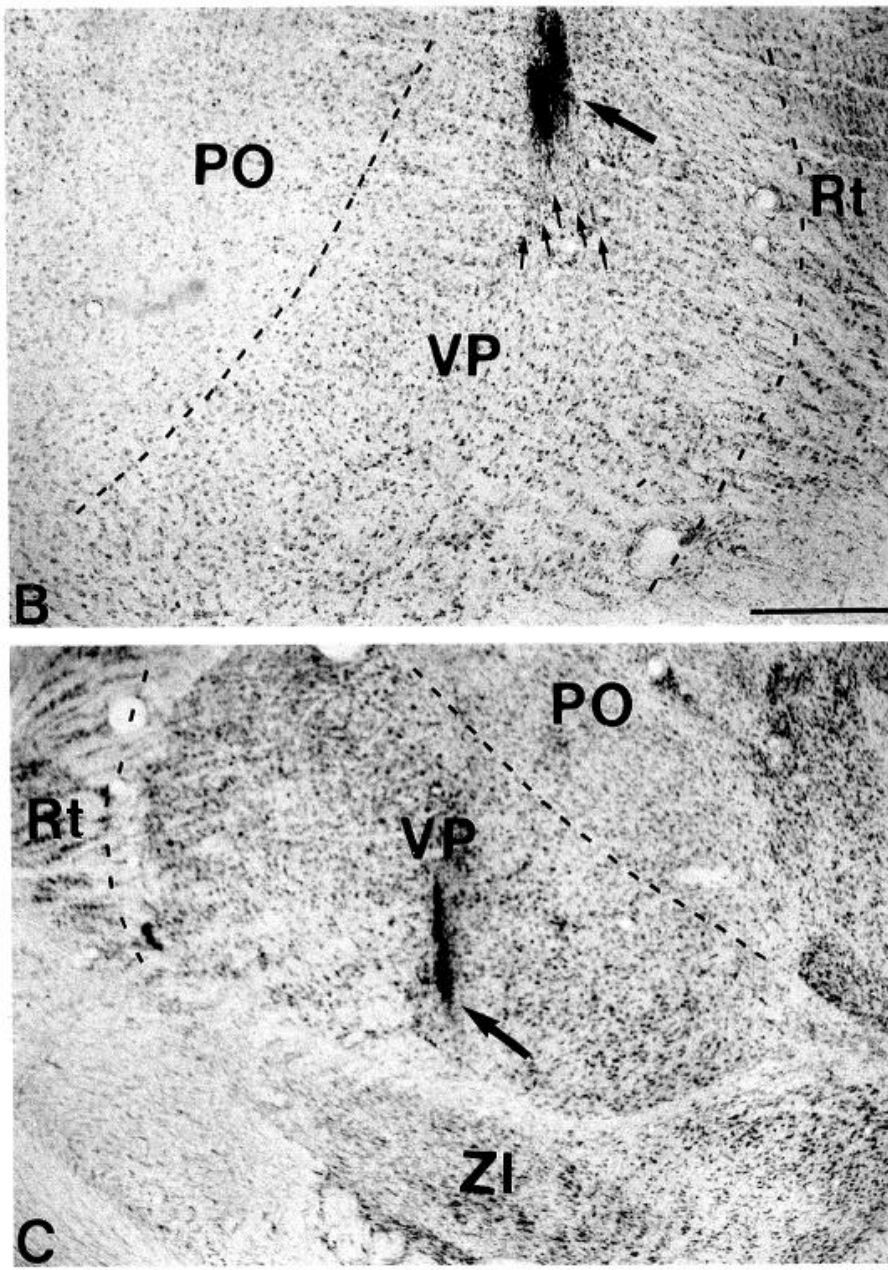

Figure 1. A, Typical $16(50 \mu \mathrm{m})$ microwire probe used in this study, as seen before the preparation required for a chronic implant (see text for details). The arrow indicates the empty pins used for EMG and ground wires. $B$ and $C$, Nissl-stained coronal sections across the VPM of two animals that received microwire bundle implants. $B$, The large arrow indicates the position of the bundle track. The position of some individual microwire tracks is indicated by small solid arrows. $C$, The position of a microwire bundle track across the VPM is indicated by a solid arrow. $P O$, posterior nucleus; $R t$, reticular nucleus; $V P M$, ventral posterior medial. Scale bars: $A$, in $\mathrm{mm} ; B, 420 \mu \mathrm{m}$ for $B$ and $C$.

suggesting that multiple single units could be recorded from each electrode.

A typical recording of a 23 neuron ensemble involved isolation of at least one neuron from each of the 16 implanted microwires and then discrimination of a second set of seven neurons from the same electrodes. A maximum of three units per microwire were statistically identified and recorded. As a rule, we first isolated the largest single unit 


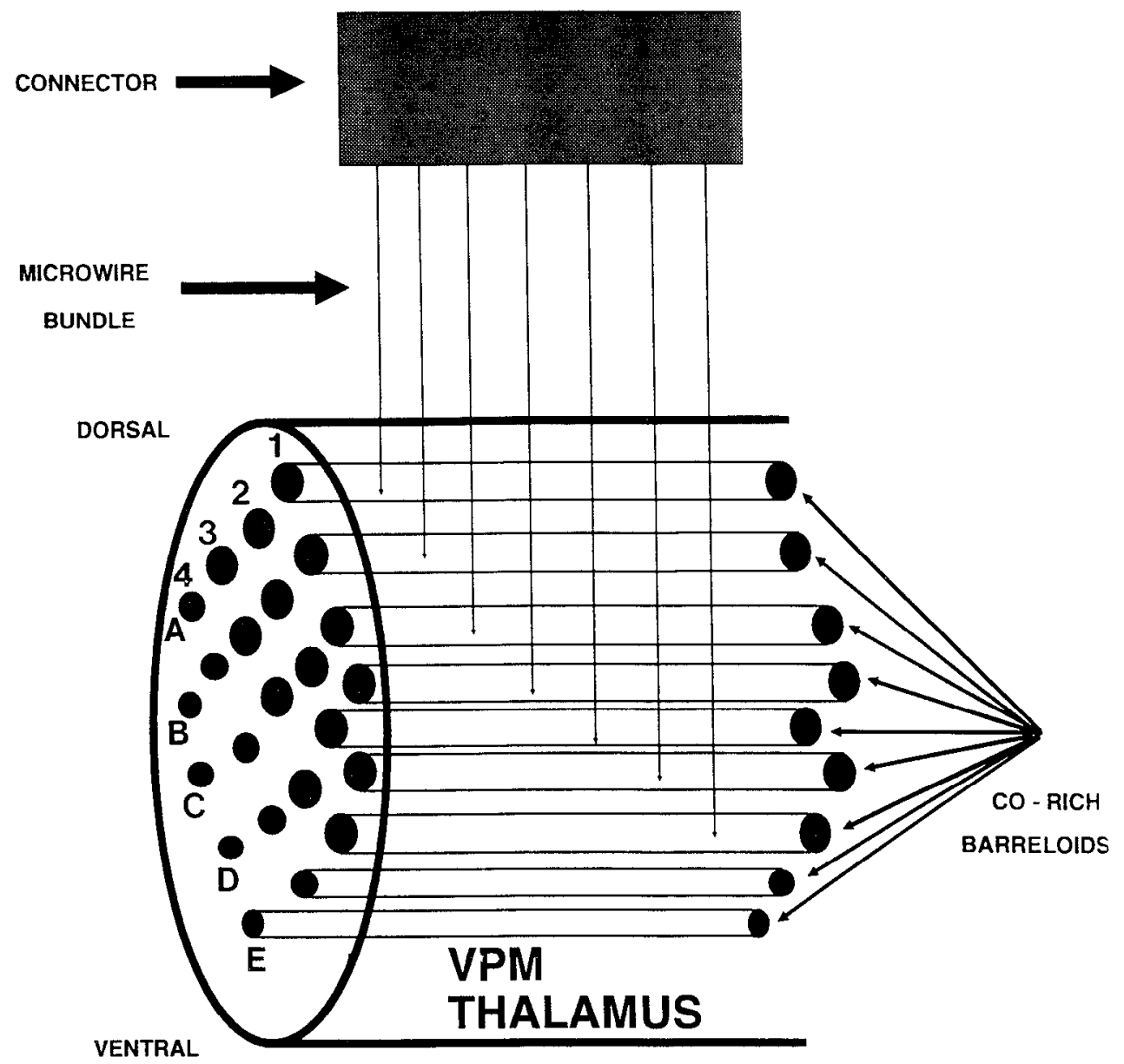

Figure 2. Representation of the recording strategy used in this study. Bundles of microwires, cut at different lengths, were implanted across multiple and distinct $\mathrm{CO}$-rich compartments of the rat VPM to allow simultaneous sampling from large numbers of single neurons.

ROSTRAL

CAUDAL

from each of the 16 microwires implanted and then attempted isolation of a second or third unit from wires that had already yielded one.

Parameters for input channel selection, amplification, filtering, and waveform discrimination were controlled by a computer, and routinely saved in configuration files. This allowed the same recording parameters to be implemented on subsequent experiments on the same animal. The outputs of the MNAP, containing the timing of single-unit firing from up to 64 channels, were transferred to a Motorola 68030 computer. MNAP outputs were first transferred to parallel input-output $(\mathrm{I} / \mathrm{O})$ boards (MVME 410, Motorola) that were then connected to 68010 MVME 104 boards installed in a Motorola 68030-based computer. Temporal series describing the timing of spiking from each isolated single neuron were continuously stored using a multitask recording software (QUNIT, Terry Bunn, Wake Forest University), designed to work under the Versados operating system. This software stored data at a time resolution of 0.2 msec and allowed simultaneous recording of up to 64 channels. Simultaneously, on-line sampling of waveforms from each channel were also carried out during the experiments (Fig. $3 C$ ). These samples were collected at the experimenter's discretion and stored by the host PC controlling the MNAP

Recording session and whisker stimulation. Prior to the recording sessions, animals were extensively habituated to the recording cage, the experimenter, and the experimental procedure (single-whisker stimulation). After initial spike discrimination, the simultaneous extracellular activity of ensembles of all discriminated neurons was continuously recorded during all stimulation experiments. A computer-controlled vibromechanical probe was used to deliver mechanical stimulation on multiple sites of the animal's face, including single facial whiskers, nose, upper and lower lip, and lower jaw. Independent repetitive stimulation of seven to nine whiskers in awake animals and up to 20 whiskers in lightly pentobarbital anesthetized animals (with blinking reflex present but no voluntary whisker movement) was carried out per recording session. For such experiments, the long, caudal whiskers were slightly trimmed on the right side of the face. Individual facial whiskers were then stimulated in a random order by positioning the probe underneath the selected whisker, $10 \mathrm{~mm}$ from the skin surface. A step pulse (100 msec duration) delivered at $1 \mathrm{~Hz}$ by a Grass $\mathrm{S} 48$ stimulator (Grass Medical Instruments, MA) was used to drive an appropriately damped small polygraph motor attached to a $10 \mathrm{~cm}$ probe. The Grass output was calibrated to produce a $0.5 \mathrm{~mm}$ whisker deflexion (initially up) and then release it. This produced an approximate $3^{\circ}$ whisker deflexion per trial. Usually 360 stimulus trials per whisker were obtained in anesthetized preparations and up to 600 trials per whisker in awake animals.

Recordings of VPM cell responses to single-whisker stimulation in awake rats were obtained after a few training sections. Usually, after a few days of preliminary recordings, and following extended running periods on the treadmill, the animals would rest in one corner of the recording cage and allow the experimenter to stimulate single whiskers repeatedly. Since good-quality single units were kept from periods varying from a few weeks to months, multiple experiments were repeated in different days in each animal.

Since both awake and anesthetized animals were used in this study, several precautions were taken to restrict stimulation to a single whisker, and avoid secondary movement of neighboring hairs. First, a portable magnification glass was used to monitor the existence of neighboring whisker movements during stimulus trials. Second, the vibromechanical stimulus was computer controlled, but could be interrupted by the experimenter at any time during the recording section. Third, EMG signals from distinct facial muscles were continuously recorded to confirm the absence of voluntary whisker movements during the stimulation procedure. Finally, the whole procedure could be taped using a high-resolution video camera, which was synchronized with the recording com- 
A
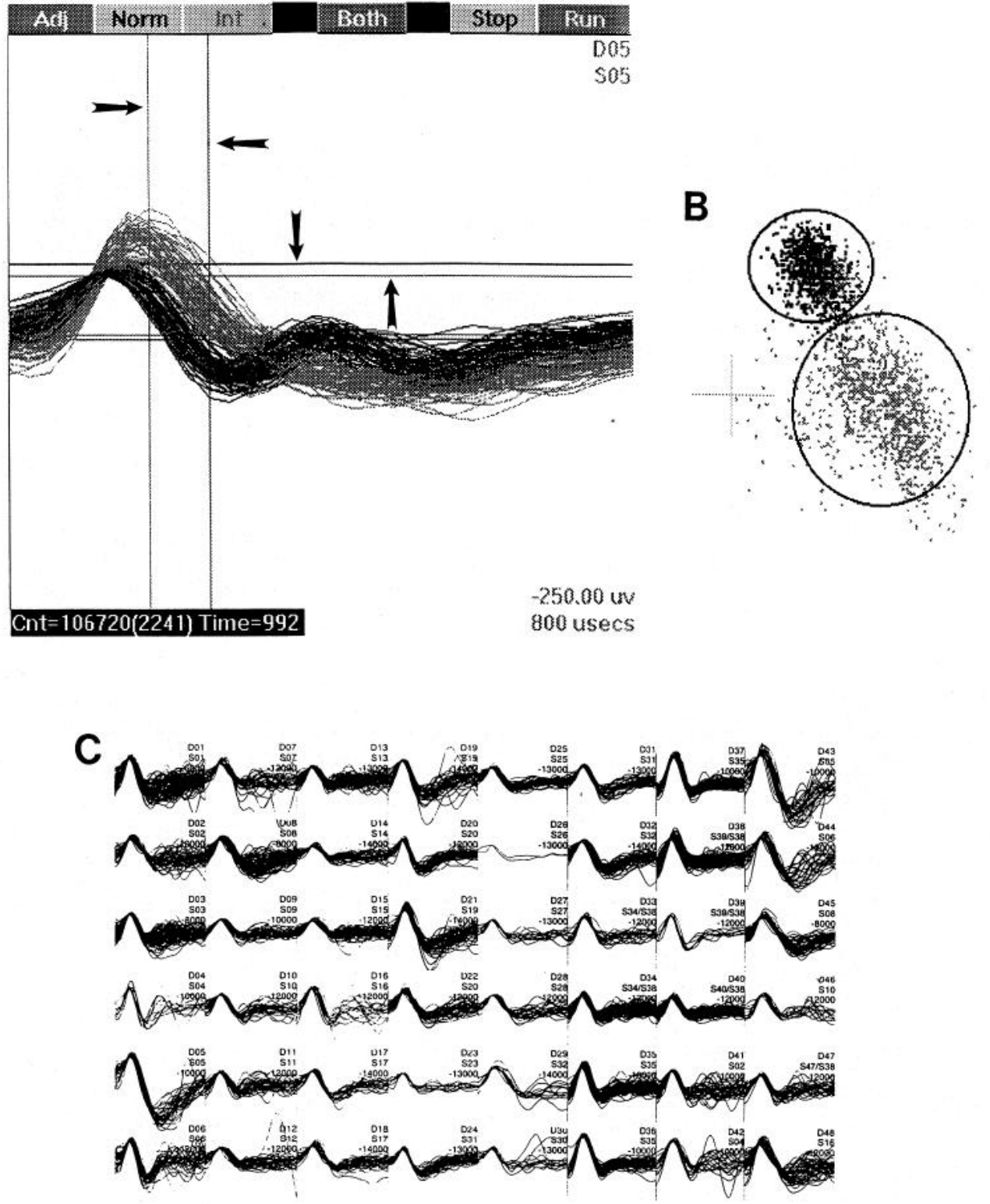

Figure 3. Discrimination of single units was obtained by combining several time-voltage windows and a realtime principal component analysis (PCA). Usually, more than one single unit was isolated from each implanted microwire. A typical example of this situation is displayed in $A$, where two waveforms (one in black and one in light gray) were isolated from the same microwire after appropriate setting of timevoltage windows (arrows). B, The corresponding clustering of waveforms obtained by PCA. $C$, Waveform sample of an experiment in which 32 thalamic cells $(D 01-D 32)$ and 16 cortical cells (D33-D48) were simultaneously recorded in an adult rat.

puter. This tape contained, on each frame, the real experimental time elapsed. This was achieved by sending a synchronizing, continuous signal from the Motorola 68030 to a video counter timer that provided a continuous time stamp (resolution $=0.1 \mathrm{sec}$ ) on the VCR tape. This procedure was particularly useful during recordings in awake animals, since it allowed elimination of time periods containing voluntary whisker or head movements.

Data analysis. Poststimulus time histograms (PSTHs) and cumulative frequency histograms (CFHs), describing each cell's response (bin width, $1-5 \mathrm{msec}$ ) to each of the whiskers stimulated, were built using an analysis program (ANALYZE). Response magnitudes (RMs), in average instantaneous spikes per second (or Hertz), were normally extracted from PSTHs during the following peristimulus epochs: $100 \mathrm{msec}$ prestimulus (control firing rate or CFR), and $3-5,5-10,10-15,15-20,20-25,25-35$, and $35-50 \mathrm{msec}$ poststimulus. The response magnitudes measured during these poststimulus epochs are represented as $\mathrm{RM}_{3-5}, \mathrm{RM}_{5-10}, \mathrm{RM}_{10-15}$, and so on.

CFHs were used to measure the latency of sensory responses. These depict cumulative poststimulus deviations from prestimulus average firing in perievent histograms. Ticks on the CFHs vertical axis represent the negative $\log$ of $p$ values describing the probability that the cumulative frequency distribution in the histogram differs from a random distribution, as computed by a one-way Kolmogorov-Smirnov (KS) test. Neuronal responses to the stimulation of a single whisker were only considered to be statistically significant if the corresponding CFHs indicated a $P<0.01$. In CFHs indicating significant sensory responses, the response latencies were recorded as the times of the inflection points in these plots, which demarcated the initial change in firing rate $(\mathrm{Ni}-$ colelis et al., 1993b; see Fig. 1). Inflection points corresponding to shortlatency responses (SLRs) and long-latency responses (LLRs) were usually identified in CFHs. The minimal latency of response $\left(\mathrm{LAT}_{\text {min }}\right.$ ) of a given cell to a single whisker was determined by the position of the first inflection point in the CFHs. Latency histograms for each simultaneously recorded VPM ensemble were built taking into account the latency values (short and long) obtained from CFHs describing all neuronal responses to all whiskers stimulated in that particular experiment.

Receptive field $(R F)$ analysis. The whisker pad is conventionally divided in dorsal to ventral rows (A-E) and caudal-to-rostral columns (16), providing each whisker with a unique name (e.g., E1). The straddle whiskers, located caudally between the rows, are named (from dorsal to ventral) $\alpha, \beta, \gamma, \delta$. RF sizes were measured in two ways. First, the total RF size for each cell was calculated by counting the absolute number of stimulated whiskers that induced a statistically significant sensory response $(P<0.01)$, as measured by the KS test performed on 


\begin{tabular}{lcccccl}
\hline Table 1. Total number of thalamic neurons recorded per animal \\
Case & Cells & Wires & $\begin{array}{l}\text { Size } \\
(\mu \mathrm{m})\end{array}$ & $\mathrm{C}: \mathrm{W}$ & $\begin{array}{l}\text { Yield } \\
(\%)\end{array}$ & RF \\
\hline MU-92 & 23 & 16 & 50 & 1.44 & 100.0 & Face \\
BU-92 & 20 & 16 & 50 & 1.25 & 68.8 & Face \\
MM-92 & 23 & 16 & 50 & 1.44 & 81.3 & Face \\
JO-92 & 19 & 16 & 50 & 1.19 & 50.0 & Face \\
GI-92 & 16 & 16 & 50 & 1.00 & 87.5 & Face \\
MI-91 & 8 & 8 & 50 & 1.00 & 100.0 & Face \\
OB-91 & 4 & 8 & 25 & 0.50 & 50.0 & Body \\
ES-91 & 8 & 8 & 25 & 1.00 & 62.5 & Neck \\
VI-91 & 8 & 8 & 25 & 1.00 & 75.0 & Face \\
VA-91 & 3 & 8 & 25 & 0.38 & 37.5 & Face \\
Total & 132 & 120 & & 1.10 & 71.3 &
\end{tabular}

The number of single cells $(\mathrm{C})$ isolated per microwire $(\mathrm{W})$ in each case is represented by the ratio $C: W$. The percentage of implanted microwires from which single units were isolated is indicated by the yield values. The general location of the receptive field (RF) of each ensemble of simultaneously recorded thalamic neurons is also indicated.

the CFHs. Total RF size was expressed in number of whiskers. For each cell, the whisker with maximum $\mathrm{RM}\left(\mathrm{RM}_{\max }\right)$ was considered the principal whisker (PW). Since for some cells the center of the RF shifted over poststimulus time, a PW was defined for both short-latency responses $\left(\mathrm{RM}_{\mathrm{s-10}}, \mathrm{PW}_{\mathrm{SL}}\right)$ and long-latency responses $\left(\mathrm{RM}_{15-35}, \mathrm{PW}_{\mathrm{LL}}\right)$. On responses $(>100 \mathrm{msec})$ were not thoroughly analyzed in this study.

The second estimation of RF size involved measurements at different magnitude thresholds of sensory-evoked responses. Five magnitude thresholds $(95 \%, 90 \%, 75 \%, 50 \%$, and $25 \%)$, representing percentages of the $\mathrm{RM}_{\mathrm{max}}$, induced by the PW, were used in this analysis. These firing thresholds were identified by the notation $\mathrm{RM}_{95 \%}, \mathrm{RM}_{90 \%}, \mathrm{RM}_{75 \%}$, and so on, and the RF size (in absolute number of whiskers) as $\mathrm{RF}_{95 \%}$,

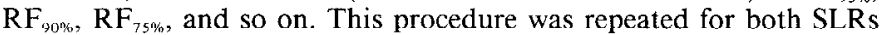
and LLRs. For each of the two populations of VPM neurons (caudalto-rostral shifting and rostral position neurons) identified in this study (see Results), RF size measurements were expressed in terms of means and standard deviations (SD). $T$ tests were used to estimate whether these two populations of VPM neurons could be distinguished in terms of RF size.

Measurement of $R F$ shifting. As described in Results, a class of VPM neurons exhibited time-dependent spatial shifts of their RF centers along the caudal-to-rostral dimension of the whisker pad. The magnitudes of these shifts were estimated for each cell by subtracting the columnar position of the $\mathrm{PW}_{\mathrm{SL}}$ from the columnar position of the $\mathrm{PW}_{\mathrm{LL}}$. This measurement was named shifting index (SFi). In this study, cells with SFi equal or greater than two whiskers were classified as exhibiting timedependent caudal-to-rostral shifts of their RFs. Cells with negative SFi $(\leq-2)$ were classified as displaying a rostral-to-caudal RF shift.

Use of factor analysis (FA) and principal component analysis (PCA) to classify spatiotemporal RF patterns. FA and PCA (Seber, 1984; Johnson and Wichern, 1988) were employed to analyze and classify the underlying functional structure of spatiotemporal RFs in the rat VPM. To classify the variety of spatiotemporal RFs observed in an ensernble, initially a raw data matrix was built in which each cell (up to 23) was treated as a variable. In these matrices, RMs indicating each cell's response to each whisker stimulated during eight consecutive poststimulus time epochs defined the cases (rows), and the cells themselves defined the columns. Thus, in an experiment in which 20 whiskers were stimulated and 23 cells were simultaneously recorded, the raw data matrix was defined by 23 columns (cells) and 160 cases (i.e., eight instantaneous firing rate samples for each of 20 whiskers stimulated). A correlation matrix was then derived from the raw data matrix by calculating the correlation coefficients between equivalent measurements from pairs of variables (cells). This matrix was then used to feed the FA/PCA subroutine of a statistical package (CSS:STATISTICA, Tulsa, OK) that derived eigenvalues, factor loadings, factor coefficients, and eigenvectors. These vectors were then subjected to a normalized varimax rotation (Seber, 1984; Johnson and Wichern, 1988). Correlated cells were then clustered in a $\mathrm{x}-\mathrm{y}$ plot whose axes were defined by the most significant factors derived. Quantitative measurements of functional association among cells during stimulation of a single whisker were obtained through the same FA/PCA procedure. This procedure was repeated for data derived from stimulation of different whiskers (different sets of eight cases) so that we could establish how functional associations among cells belonging to the simultaneously recorded ensemble varied according to the whisker stimulated.

Graphical analysis of single-cell and population spatiotemporal $R F$. Spatial and temporal attributes of single-cell and population RFs were evaluated through 3-D and 4-D graphic representations constructed through use of three commercially available software packages: GRAFTOOL (3-D Visions Corp.), css:statistica (Statsoft, Tulsa, OK), and simulinK (The Math Works Inc., Natick, MA). RM values derived from PSTHs were used as raw data for all such representations.

Changes in single-cell RFs over poststimulus time were first depicted in sequences of 3-D graphs, each representing the distribution of the magnitude of sensory-evoked responses of a VPM neuron as a function of the spatial location of the stimulated whiskers. The whisker pad structure was reproduced by representing the five ventral to dorsal whisker rows (A-E), separated by four single "straddle" whiskers $(\alpha, \beta, \gamma, \delta)$, in the $\mathrm{x}$-axis, while the $\mathrm{y}$-axis (left horizontal axis) represented the column position of whiskers within the rows (I, for most caudal whiskers; V, for most rostral). The z-axis (vertical) represented the cell's instantaneous RM for each stimulated whisker. A fourth dimension, poststimulus time, was obtained by plotting a continuous sequence of six such graphs covering time epochs from $5-10 \mathrm{msec}$ to $35-50 \mathrm{msec}$. Statistical differences in spatial distributions of sensory responses at different time epochs were evaluated using a $\chi^{2}$ test and analysis of variance (ANOVA).

To cxpress these temporal and spatial data in one graph, the 2-D whisker location coordinates were collapsed into one dimension. These "spatiotemporal RFs" depicted the cell's RM (z-axis) as a function of stimulus location (whisker position, $\mathrm{x}$-axis) and poststimulus time (milliseconds, y-axis). This involved a caudal-to-rostral rank ordering of the stimulated whiskers (usually involving a whisker row). To improve visualization of the continuous spatiotemporal structure of the cell's RF, experimental data were then smoothed by a spline algorithm based on a third-order polynomial function (Johnson and Ries, 1982), using a very low "stiffness" coefficient to reduce the impact of outliers, while expressing local changes thoroughly. The resulting 3-D surface was then color coded in eight categories of RM (z-axis) ranging from red (highest RM) to blue (lowest). Statistical significance of instantaneous sensoryevoked responses was verified through analysis of variance (ANOVA) associatcd with a Dunnett's post hoc test.

Two complementary population RF representations were employed here. Population PSTHs described the sensory response of a neuronal ensemble to the stimulation of a single whisker as a function of poststimulus time. To build these graphs, cells were first rank ordered according to their $\mathbf{R M}_{\max }$. Next, the simultaneous recorded sensory responses were plotted together in a 3-D graph. The $x$-axis of this graph represented poststimulus time in milliseconds; the y-axis, the cell rank order; and the z-axis, the RM. A series of population PSTHs (one for each stimulated whisker) was built for each ensemble. Population PSTHs were complemented by spatiotemporal population RFs. These illustrated the ensemble RM as a function of multiple stimulus locations in a given poststimulus epoch. To build these latter representations, cells were first rank ordered ( $\mathrm{x}$-axis). Then, their RMs ( $\mathrm{z}$-axis), at a given poststimulus epoch, werc plottcd as a function of the stimulus location (y-axis). A spline algorithm (css:STATISTICA) or another interpolation routine (SIMULINK) was used to fit a color-coded 3-D surface on the original scatter plot. A fourth dimension, poststimulus time, was then obtained by plotting series of these graphs.

Histology. Microwire location was confirmed through analysis of Nisslstained sections. In some cases, electrical lesions in the VPM were produced in deeply anesthetized animals by passing current (5-10 $\mu \mathrm{A}$ for $15-20 \mathrm{sec}$ ) through one or two microwires. Since the microwires remained in the brain for long periods of time, electrode tracks and tip positions could be identified by small but detectable glia scars, even without electrical lesions. After the completion of recordings, animals were deeply anesthetized with pentobarbital $(100-200 \mathrm{mg} / \mathrm{kg})$ and then perfused intracardiacally, first with $0.9 \%$ saline solution $(5-10 \mathrm{~min}$ ) followed by a solution of $4 \%$ formalin in $0.9 \%$ saline ( $10-15 \mathrm{~min})$. Brains were kept for $24-48 \mathrm{hr}$ at $4^{\circ}$ in $30-40 \%$ sucrose dissolved in the same fixative solution. Coronal brain sections $(80 \mu \mathrm{m})$ were obtained in a freezing microtome and mounted in gel-coated slides, air dried, stained for Nissl, and coverslipped. Microwire tracks (see Fig. $1 B, C$ ) and tips were located using a Nikon microscope. 

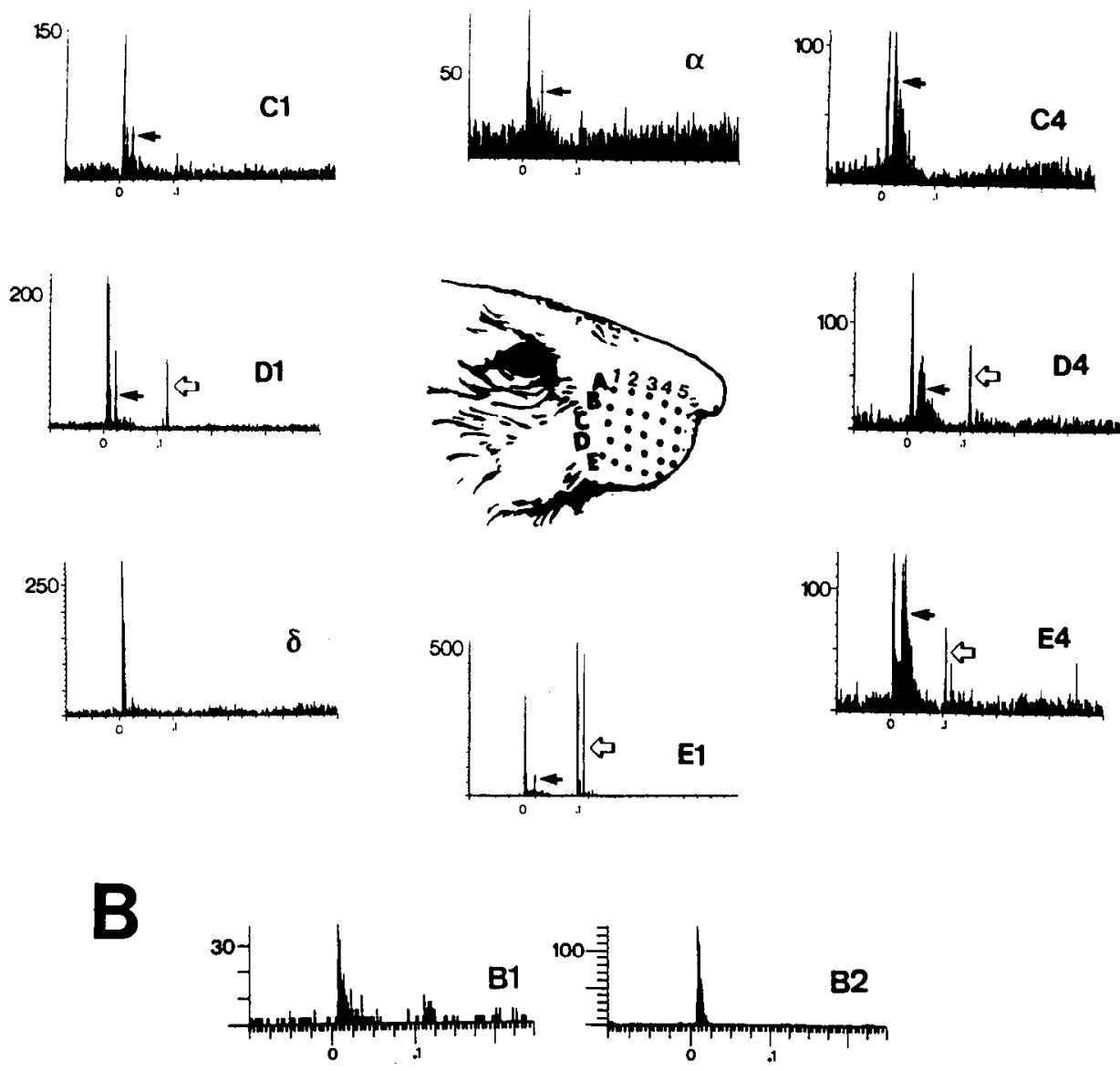

B2
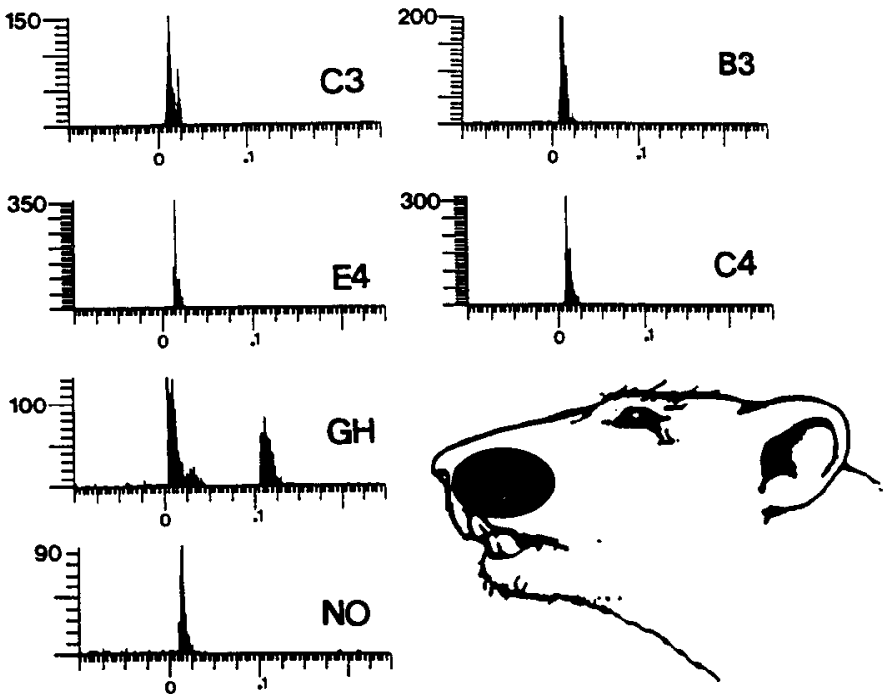

Figure 4. Multiwhisker RFs in the VPM of a lightly anesthetized adult rat. In $A$, the $\mathrm{RF}$ of a single $\mathrm{C} \rightarrow \mathrm{R}$ shifting VPM cell included whisker $E$ I (RF center), but also several other whiskers located in different rows and columns. As the stimulus moved away from E1, the magnitude of short-latency responses decayed. Brief short-latency excitatory responses were followed by inhibitory and then long-latency excitatory responses (solid arrows) that were more noticeable when the stimulus was delivered in the RF surround. Off responses are indicated by open arrows. A drawing of the rat's face represents the distribution of whiskers in rows $(A-$ $E$ ) and columns (1-5). $B$, An RP cell with RF centered in rostral whiskers also exhibited large RFs (smaller than $C \rightarrow R$ cells) that could span entire whisker rows, guard hairs $(G H)$, and even reach the nose $(N O)$. Likewise in $A$, these cells exhibited a decay in response magnitude as the stimulus moved away from the RF center. Nevertheless, isolated long-latency responses were more rarely observed. A drawing of the rat's face indicates the extent of this cell's RF. In both $A$ and $B$. PSTHs are based on 360 trials obtained with an $100 \mathrm{msec}$ duration stimulus delivered at $1 \mathrm{~Hz}$. Time is in seconds $(0=$ stimulus onset; $0.1 \mathrm{sec}=$ stimulus offset).

\section{Results}

Ten adult rats (Table 1) provided a total sample of 132 cells, obtained from 120 microwires implanted in the VP complex. The yield, that is, the percentage of the total number wires from which single units could be recorded, was $71.3 \pm 21.5 \%$ (mean $\pm \mathrm{SD}$ ).

\section{Awake versus anesthetized recordings}

RF properties and sensory response latencies of VPM neurons were found to be qualitatively similar under both awake and lightly anesthetized conditions. On the other hand, the spontaneous firing rates were significantly higher in awake (mean \pm $\mathrm{SEM}, 7.13 \pm 0.31 \mathrm{~Hz}$ ) than in anesthetized (mean $\pm \mathrm{SEM}, 2.83$ $\pm 0.11 \mathrm{~Hz}$ ) conditions and the overall complexity of the sensory response patterns was also greater. Because independent stimulation of all whiskers was not possible in awake rats, makin£ statistical classification of RFs more difficult, most of the following quantitative description of results is based on data ob. tained in lightly anesthetized animals in which up to 20 single whiskers were independently stimulated. 
Table 2. Differences in RF size between caudal-to-rostral $(C \rightarrow R)$ shifting neurons and rostral position $(\mathrm{RP})$ cells

\begin{tabular}{|c|c|c|c|c|c|c|c|c|c|c|c|c|}
\hline \multirow[b]{3}{*}{ Class } & \multirow{3}{*}{$\begin{array}{l}\text { RF } \\
(W)\end{array}$} & \multicolumn{6}{|l|}{ SLRs } & \multicolumn{5}{|l|}{ ILLRs } \\
\hline & & \multirow[b]{2}{*}{$\mathrm{RM}(\mathrm{Hz})$} & \multicolumn{5}{|l|}{ RF } & \multirow[b]{2}{*}{$\mathrm{RM}(\mathrm{Hz})$} & \multicolumn{4}{|l|}{ RF } \\
\hline & & & $95 \%$ & $90 \%$ & $75 \%$ & $50 \%$ & $25 \%$ & & $95 \%$ & $90 \%$ & $75 \%$ & $50 \%$ \\
\hline Total & 15.27 & 119.63 & 1.41 & 1.76 & 3.07 & 5.17 & 7.85 & 47.49 & 1.17 & 1.37 & 1.93 & 4.29 \\
\hline$(n=41)$ & 3.91 & 50.12 & 0.71 & 1.04 & 1.77 & 3.05 & 4.00 & 33.34 & 0.44 & 0.62 & 1.13 & 2.67 \\
\hline \multicolumn{13}{|l|}{$\mathrm{C} \rightarrow \mathrm{R}$} \\
\hline cells & 17.15 & 131.68 & 1.53 & 1.95 & 3.47 & 6.37 & 9.32 & 46.00 & 1.16 & 1.37 & 2.00 & 4.74 \\
\hline$(n=19)$ & 2.43 & 63.48 & 0.70 & 1.08 & 1.58 & 2.56 & 3.30 & 33.57 & 0.37 & 0.60 & 1.29 & 3.19 \\
\hline \multicolumn{2}{|l|}{ Position } & 109.81 & & 1.25 & 2.13 & 3.13 & 5.50 & 48.50 & 1.19 & 1.25 & 1.69 & 3.75 \\
\hline$(n=16)$ & 4.05 & 26.63 & 0.52 & 0.59 & 0.96 & 1.85 & 3.27 & 31.64 & 0.26 & 0.35 & 0.64 & 2.32 \\
\hline$P$ & $<9 \mathrm{E}-4$ & & $<0.03$ & $<0.01$ & $<2 \mathrm{E}-3$ & $<7 \mathrm{E}-5$ & $<7 \mathrm{E}-4$ & & NS & NS & NS & NS \\
\hline
\end{tabular}

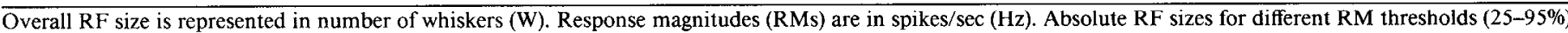

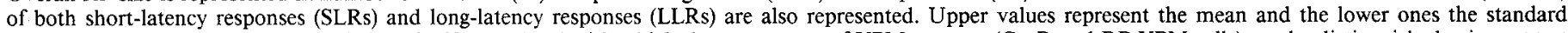

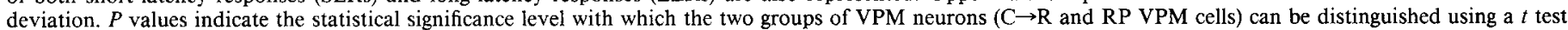
for each of the RF measurements. NS, non-significant.

\section{Quantitative measurement of receptive field sizes in the VPM}

In general, quantitative and statistical analysis (see Materials and Methods) of PSTHs and CFHs revealed that VPM neurons have large, multiwhisker RFs. Of the 120 recorded cells, 82 (68.3\%) exhibited RFs centered in the whisker pad and/or in surrounding regions (guard hairs, upper lip, nose, lower lip) and responded strongly to low-threshold mechanical stimulation of these sites. Histological analysis of microwire tracks verified that these units were located in the VPM (Fig. $1 B, C$ ). The remaining 38 cells, which were not further analyzed, had RFs centered in other facial skin territories (caudal face, neck, lower jaw; from wires in the lateral and ventral VPM), other body regions (forepaw and hindpaw; from wires in the VPL), or responded only to stimulation of higher threshold or proprioceptors (from wires in the dorsal cap of the VPM).

In our sample of 82 VPM cells, $55(67 \%)$ RFs were centered mainly on the whisker pad and $27(33 \%)$ were centered on guard hairs, upper lip, and/or nose. Among the 55 cells with RFs centered on the whisker pad, $51(93 \%)$ yielded statistically significant $(P<0.01)$ responses to the stimulation of six or more independent whiskers. Forty $(73 \%)$ responded to 10 or more whiskers and $34(64 \%)$ to 15 or more whiskers. The average RF for this sample was $13.7 \pm 4.8$ (mean $\pm \mathrm{SD}$ ) whiskers.

As an example, Figure 4 illustrates two distinct categories of RFs observed in the VPM (" $C \rightarrow R$ " and "RP" cells, named according to a complex set of criteria described below). The RF represented in Figure $4 A(\mathrm{C} \rightarrow \mathrm{R}$ cell $)$ included all 20 whiskers stimulated in the experiment. Its short-latency principal whisker $\left(\mathrm{PW}_{\mathrm{SI}}\right)$ was E1. Its minimal latency $\left(\mathrm{LAT}_{\min }\right)$ was 4 msec. Its response magnitude in the $5-10 \mathrm{msec}$ poststimulus epoch $\left(\mathrm{RM}_{5-10}\right)$ was $210 \mathrm{~Hz}$. As the stimulus moved to the surrounding whiskers, smaller but highly significant responses were observed $(P<0.01)$. As is typical of VPM cells, this neuron displayed robust but brief short-latency responscs (SLRs) whose LAT $_{\text {min }}$ ranged from 4 to $10 \mathrm{msec}$ after the stimulus. These excitatory responses were typically followed by brief postexcitatory inhibitions and then by excitatory long-latency responses (LLRs), which themselves were followed by a second inhibitory response. The $\mathrm{RM}_{5-10}$, which provides the best standardized measurement of the magnitude of the SLRs, decayed up to 10-fold from the cell's RF center (whisker El) to its far excitatory surround (whisker $\alpha$ ) where highly significant responses were still obtaincd. The magnitudes of LLRs (see black arrowheads in Fig. $4 A$ ) also varied according to whisker position and poststimulus time. Off responses (Fig. $4 A$, open arrows, after 100 msec) also exhibited short- and long-latency components. However, whisker stimulation did not always induce off responses. This is consistent with previous reports of direction selectivity in the VPM (Waite, 1973b; Simons and Carvell, 1989).

Figure $4 B$ (RP cell) shows a VPM RF centered in the rostral face $\left(\mathrm{PW}_{\mathrm{SL}}=\mathrm{C} 4, \mathrm{RM}_{5-10}=167 \mathrm{~Hz}, \mathrm{LAT}_{\min }=8 \mathrm{msec}\right)$. This cell's RF was somewhat smaller ( 12 whiskers) but involved not only whiskers throughout the caudorostral axis of the face (from $\mathrm{B} 1$ to E4) but also a guard hair $(\mathrm{GH})$ and the lateral edge of the nose. The RMs decayed approximately 10 -fold from the center of the RF (C4) to the far surround (B1). LLRs and off responses were weaker in this cell. Long-lasting excitatory responses, beginning from 4 to $8 \mathrm{msec}$ and ending from 15 to $25 \mathrm{msec}$ poststimulus time, dominated the profile of the overall sensory response.

\section{Defining RF topology}

Because the characteristic topology of VPM RFs included "dropoffs" from center to surround, one can define a different RF size for distinct thresholds of response magnitude (RM). Therefore, for each cell we also measured RF size as a function of a series of response magnitude thresholds, expressed as percentages $(25 \%$ 95\%) of the $\mathrm{RM}_{\max }$ obtained by stimulating the PW. Table 2 contains the results of this analysis. For a sample of 41 VPM cells, whose $\mathrm{PW}_{\mathrm{SL}}$ was definitively identified, the $\mathrm{RM}_{\max }$ at 510 msec averaged $119.63 \pm 50.12$ (mean \pm SD) $\mathrm{Hz}$. The average size of RFs at the $\mathrm{RM}_{95 \%}$ was $1.41 \pm 0.70$ (mean $\pm \mathrm{SD}$ ) whiskers. Therefore, $\mathbf{R F}_{95 \%}$ was shown to be roughly equivalent to the principal whisker or RF center. As the measured RM threshold chosen for this analysis was reduced, the mean RF size increased so that at $\mathrm{RM}_{50 \%}$ the mean $\mathrm{RF}$ size was $5.17 \pm 3.05$ (mean \pm SD) whiskers, and at $\mathrm{RM}_{25 \%} 7.85 \pm 4.00$ (mean $\pm \mathrm{SD}$ ) whiskers.

The variation of short-latency RM (from 4 to $10 \mathrm{msec}$ ) as a function of LAT $_{\min }$ was also estimated for each VPM cell. No linear correlation was found between the RM and $\mathrm{LAT}_{\min }$ (Arm- 


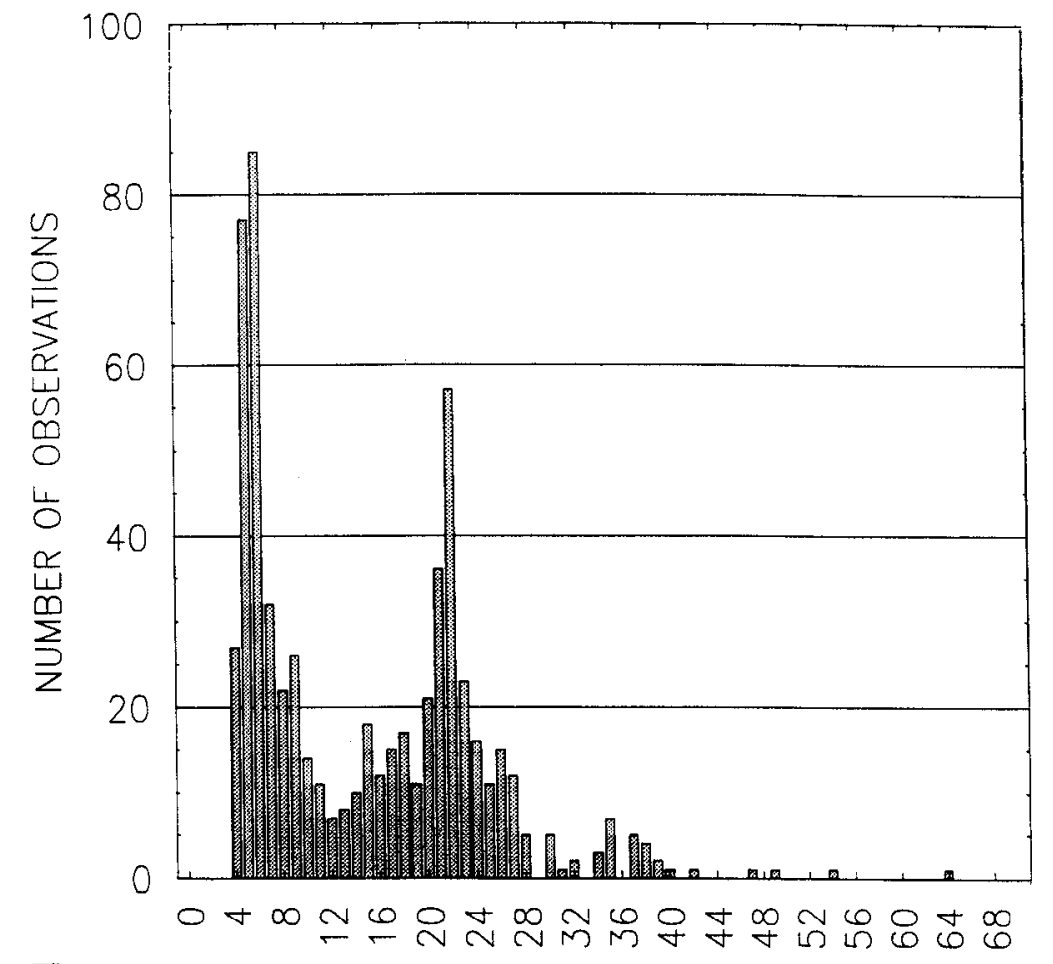

$\mathbf{A}$

LATENCY (ms)

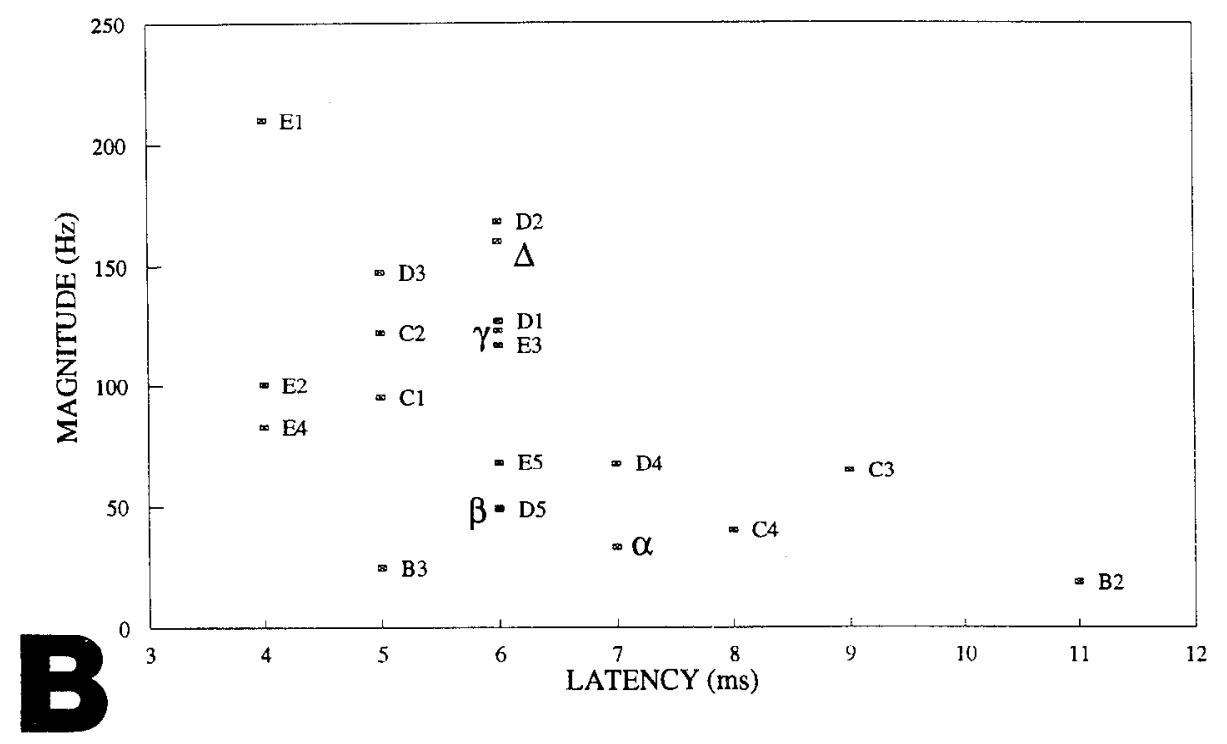

Figure 5. Latency distribution and relationship between magnitude and latency of sensory responses. $A$, This bimodal latency distribution obtained from 23 simultaneously recorded VPM neurons demonstrates that VPM sensory responses were formed by shortlatency (mean $\pm \mathrm{SD}, 6.4 \pm 1.83 \mathrm{msec})$ and long-latency excitatory components (mean $\perp \mathrm{SD}, 21.78 \perp 6.42 \mathrm{msec}$ ). $B$, Scatter plot of the magnitude of sensory responses of a single VPM cell as a function of the minimal response latency, following the independent stimulation of 20 whiskers. Notice the considerable temporal overlap between sensory responses obtained by stimulation of the RF center (E1), near surround $(\gamma, \delta, \mathrm{C} 2, \mathrm{D} 1, \mathrm{D} 2, \mathrm{D} 3, \mathrm{E} 3)$, and far surround $\operatorname{RF}(\alpha, \beta, \mathrm{B} 2, \mathrm{~B} 3, \mathrm{C} 1, \mathrm{C} 3$, C4, D4, D5, E2, E4, E5).
strong-James and Callahan, 1991), demonstrating that spatial discrimination in the VPM does not depend exclusively on linear differences in response latency. Figure $5 B$ illustrates one example in which the short-latency responses of a VPM cell, elicited by independent stimulation of 19 whiskers, was plotted as a function of $\mathrm{LAT}_{\min }$. For this cell, whisker E1 defined the $\mathrm{PW}_{\mathrm{SL}}$. The $\mathrm{LAT}_{\min }$ for this whisker was $4 \mathrm{msec}$. A second group of seven whiskers $(\gamma, \delta, \mathrm{C} 2, \mathrm{D} 1, \mathrm{D} 2, \mathrm{D} 3, \mathrm{E} 3)$ defined the immediate or near RF excitatory surround $(50 \%<\mathrm{RM}>95 \%)$. Their responses occurred in a range of $5-7 \mathrm{msec}$. A far surround
$(\mathrm{RM}<50 \%)$ defined by 11 whiskers $(\alpha, \beta, \mathrm{B} 2, \mathrm{~B} 3, \mathrm{C} 1, \mathrm{C} 3, \mathrm{C} 4$, $\mathrm{D} 4, \mathrm{D} 5, \mathrm{E} 2, \mathrm{E} 4, \mathrm{E} 5)$ induced sensory responses in the range of 4-11 msec. Overall, stimulation of the RF center induced the earliest sensory response (mean $\pm \mathrm{SD}, 5.1 \pm 1.1 \mathrm{msec}$, range 4-8 $\mathrm{msec}$ ). Stimulation of the immediate RF surround induced lowe ${ }_{i}$ RMs, and a $\mathrm{LAT}_{\min }$ (mean $\pm \mathrm{SD}, 5.5 \pm 0.8 \mathrm{msec}$, range 4-8 $\mathrm{msec}$ ) that overlapped considerably with that of the RF center. Stimulation of the RF far surrounds induced responses at a slightly higher $\mathrm{LAT}_{\text {min }}$ (mean $\pm \mathrm{SD}, 6.4 \pm 1.6$ msec, range 4-11 msec). 
Table 3. Drop-off in response magnitude (RM, in $\mathrm{Hz}$ ) across whisker rows defined by SLRs of six $C \rightarrow R$ shifting and six RP cells $(C)$

\begin{tabular}{|c|c|c|c|c|c|c|}
\hline & $\mathrm{CO1}$ & $\mathrm{CO} 2$ & $\mathrm{CO3}$ & $\mathrm{CO} 4$ & $\cos$ & $\mathrm{C06}$ \\
\hline \multicolumn{7}{|c|}{$\mathrm{C} \rightarrow \mathrm{R}$ shifting cells } \\
\hline El & 163 & 210 & 74 & 98 & 223 & 83 \\
\hline $\mathrm{E} 2$ & 168 & 100 & 57 & 50 & 212 & 64 \\
\hline E3 & 123 & 117 & 34 & 23 & 134 & 25 \\
\hline E4 & 46 & 83 & 11 & 15 & 21 & 29 \\
\hline \multirow[t]{4}{*}{ E5 } & 19 & 68 & 7 & 10 & 10 & 18 \\
\hline & $a=-41$ & $a=-30$ & $a=-18$ & $a=-21$ & $a=-62$ & $a=-17$ \\
\hline & $r=0.95$ & $r=0.85$ & $r=0.98$ & $r=0.91$ & $r=0.96$ & $r=0.92$ \\
\hline & $P<0.002$ & $P<0.01$ & $P<0.0007$ & $P<0.01$ & $P<0.002$ & $P<0.005$ \\
\hline
\end{tabular}

RP cells

$\begin{array}{rrrrrr}110 & 108 & 116 & 63 & 167 & 107 \\ 51 & 91 & 23 & 9 & 139 & 62 \\ 6 & 4 & 8 & 1 & 86 & 8 \\ 3 & 37 & 4 & 0 & 23 & 0 \\ 4 & 44 & 9 & 1 & 9 & 1\end{array}$

For each $\mathrm{C} \rightarrow \mathrm{R}$ cell, the top number corresponds to the RM obtained by stimulating E1. Subsequent values correspond to the RM obtained for the other four whiskers in the E row (E2, E3, E4, and E5). For RP cells, the first RM value corresponds to the rostral whisker that defined the cell's $\mathrm{PW}$ stimulating more caudal whiskers. $a=$ slope, $r=$ correlation coefficient for linear fitting, and $P=$ significance level.

\section{The $R F$ size defined by different latency components}

As mentioned above, thalamic cells displayed SLRs (range 410 msec poststimulus) and/or LLKs (15-25 msec poststimulus). The distribution of response latencies of VPM neurons to center and surround stimulation was clcarly bimodal. As an cxamplc, Figure $5 A$ shows the latency distribution of responses from a simultaneously recorded ensemble of 23 cells to independent stimulation of 20 whiskers. These latency measurements were derived from analysis of 460 CFHs. The onset of SLRs occurred mainly between 4 and 6 msec poststimulus (mean $\pm \mathrm{SD}, 6.4$ $\pm 1.8 \mathrm{msec}$ ), though they could be observed at latencies up to 10 msec. The onset of LLRs, on the other hand, occurred at a minimum of $15 \mathrm{msec}$, peaked around 20-24 msec, and occasionally were observed up to $40 \mathrm{msec}$ (mean $\pm \mathrm{SD}, 21.78 \pm$ $6.4 \mathrm{msec}$ ).

LLRs were invariably weaker (mean $\pm \mathrm{SD}, 47.49 \pm 33.34$ $\mathrm{Hz}$ ) than SLRs (mean $\pm \mathrm{SD}, 119.63 \pm 50.12 \mathrm{~Hz}$ ). Nonetheless, excitatory LLRs tended to define smaller RFs than SLRs at different RM thresholds (Table 2). Furthermore, of the 55 VPM neurons with RFs located exclusively on the whisker pad, 23 (41.8\%) had different RF centers defined by the LLRs (the

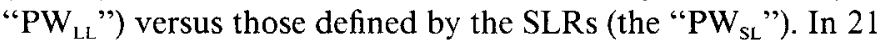
of these cells, the $\mathrm{PW}_{\mathrm{LL}}$ was located in a more rostral whisker than the $\mathrm{PW}_{\mathrm{SL}}$. These caudal-to-rostral $(\mathrm{C} \rightarrow \mathrm{R})$ shifts of $\mathrm{RF}$ centers were observed in $100 \%$ of the cells $(n=12)$ whose $\mathrm{PW}_{\mathrm{SL}}$ was located in the first column of the whisker pad (A1-E1). $\mathrm{C} \rightarrow \mathrm{R}$ shifts occurred in $80 \%$ of the cells whose $\mathrm{PW}_{\mathrm{SL}}$ was located in the second column $(n=5)$ and $80 \%$ of the straddle whiskers $(n=5)$ but were rare $(14.3 \%)$ in cells with $\mathrm{PW}_{\mathrm{SL}}$ in the third column $(n=7)$. Neurons exhibiting this type of dynamic RF change were collectively designated as caudal-to-rostral $(C \rightarrow R)$ shifting cells. By definition, these neurons exhibited a positive shifting index (SFi).

This $\mathrm{C} \rightarrow \mathrm{R}$ RF shifting property was generally found in VPM neurons with the largest RFs (considering SLRs) of our sample (mean $\pm \mathrm{SD}, 17.2 \pm 2.4$ whiskers). Though RF centers and surrounds at short latency were large, the RFs of these neurons at long latency were relatively smaller (Table 2 ). These cells also tended to exhibit a linear drop-off in short-latency response magnitude over whisker rows (Table 3). In a sample of seven cells whose RFs contained the E row, the average RM drop-off per whisker (from E1 to E5) was $32.71 \pm 16.26 \mathrm{~Hz}$ (mean \pm SD). However, RMs of the LLRs did not drop off linearly from the $\mathrm{PW}_{\mathrm{LL}}$, but instead exhibited a more circumscribed appearance.

The spatiotemporal complexity of these continuous, timedependent $\mathrm{C} \rightarrow \mathrm{R}$ shifts can be better visualized in 3-D graphs such as in Figure 6. During the $5-10 \mathrm{msec}$ poststimulus epoch, the RF of this cell decayed linearly from its RF center (E1 whisker) to cover most of the animal's whisker pad. By 10-15 msec, however, the $\mathrm{RM}_{\text {max }}$ was no longer produced by $\mathrm{E} 1$, but by D2. At 15-20 $\mathrm{msec}$, the strongest responses have shifted toward more rostral territories, such that by $25-35 \mathrm{msec}$ the $\mathrm{PW}_{\mathrm{LL}}$ was the E4 whisker. Thus, over a period of $25 \mathrm{msec}$, a time-dependent $\mathrm{C} \rightarrow \mathrm{R}$ of this cell's RF center was gradually

Figure 6. Spatiotemporal RF of a caudal-to-rostral $(\mathrm{C} \rightarrow \mathrm{R})$ shifting VPM cell. In this 4-D RF representation, each 3-D graph depicts the spatial domain of the cell's RF at a given poststimulus time interval (identified in the upper right corner of each 3-D figure). Each vertical bar in these 3-D graphs depicts the average instantaneous RM of a VPM neuron following repetitive stimulation of a single whisker. The overall distribution of these bars defines the spatial domain profile of the RF at each time epoch. For this cell, the RF center defined by SLRs (represented by the 3-D graph at 5-10 msec) was whisker E1. Over subsequent poststimulus time, this RF center gradually shifted to a more rostral position so that at $25-$ $35 \mathrm{msec}$ a complete caudal-to-rostral shift was established. Caudal whiskers that formed the center and near surround at short latency passed to define the long-latency RF far surround. 

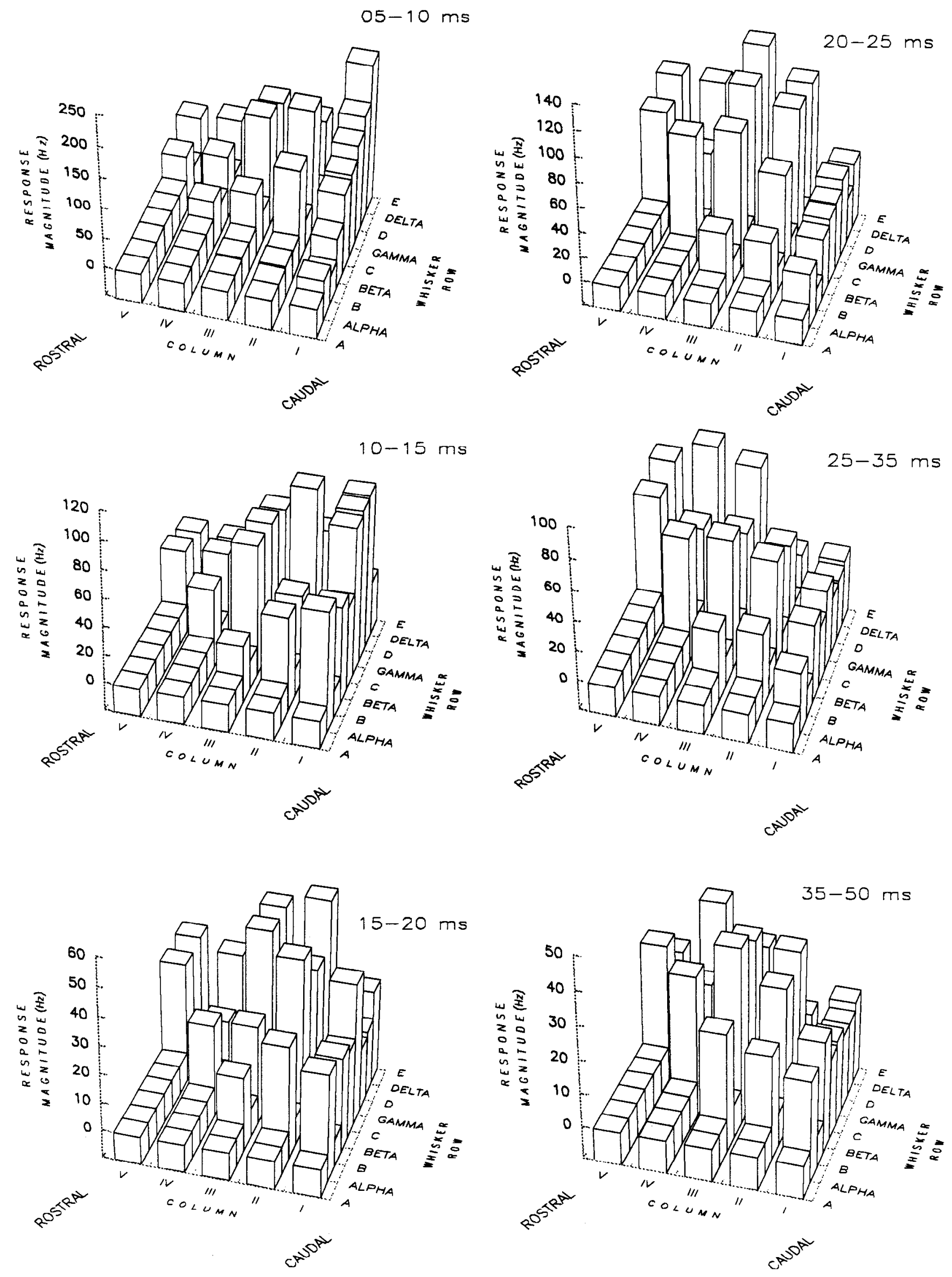

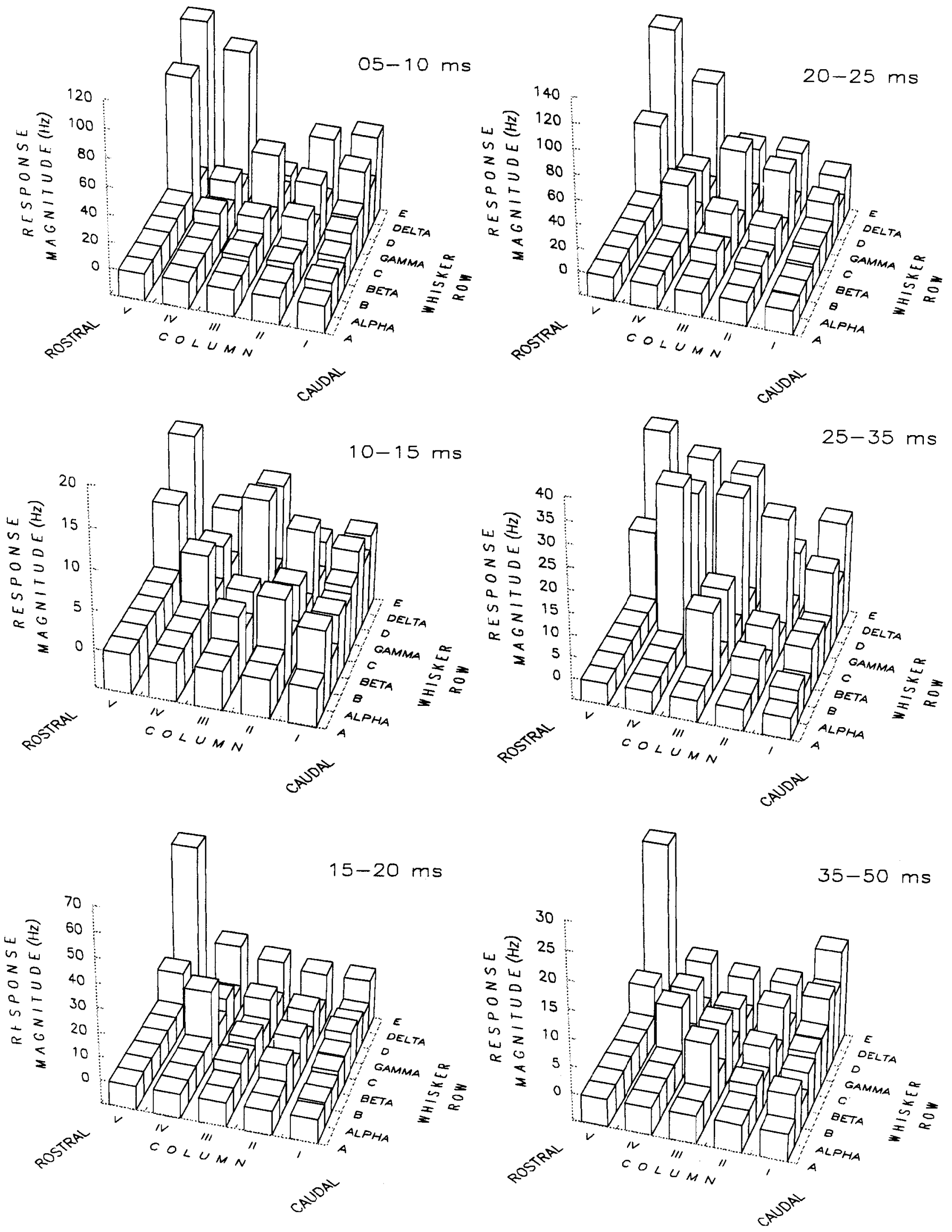
established. This appeared to involve a combination of two factors: (1) a persistent in-field inhibition of the short-latency RF center and its immediate surround, and (2) a time-dependent enhancement of sensory-evoked responses obtained from rostral whiskers. Further analysis of this cell's spatiotemporal RF (see Fig. $11 \mathrm{~A}-\mathrm{C}$ ) by whisker row revealed that a greater caudal-torostral RF shift occurred across row $\mathrm{E}$ than across rows $\mathrm{D}$ and C. Figure $1 \mathrm{l} A$ also illustrates the fact that this cell's SLRs exhibited a clear linear RM drop-off across the E row.

As a rulc, $C \rightarrow R$ shifts were never observed in cclls with $P W_{S L}$ located in higher columns (4-6). For 11 of the cells with $\mathrm{PW}_{\mathrm{SL}}$ centered in columns $4-6(n=12)$, the position of the $\mathrm{PW}_{\mathrm{LL}}$ was either identical to the $\mathrm{PW}_{\mathrm{SL}}$ or included a neighboring whisker in the same column of the $\mathrm{PW}_{\mathrm{SL}}$. In addition, most of the cells with $\mathrm{PW}_{\mathrm{SL}}$ in the third column did not exhibit RF shifts. VPM neurons that did not exhibit RF center shifts were collectively designated rostral position (RP) cells. The RF sizes defined by their SLRs and LLRs were identical (Table 2). These VPM neurons had smaller overall RFs (mean \pm SD, 13.1 \pm 4 . 1 whiskers) than $C \rightarrow R$ shifting cells and smaller short-latency $R F$ sizes. However, no statistical difference in long-latency RF size was observed between $\mathrm{C} \rightarrow \mathrm{R}$ shifting and RP cells. Generally, RP neurons exhibited more exponential decays in short-latency $R M$ from the RF center to its surrounds (Table 3).

Figure 7 illustrates the RF of one of these RP neurons. Its $\mathrm{PW}_{\mathrm{SL}}$ was whisker E5 $\left(\mathrm{RM}_{\max }=109 \mathrm{~Hz}\right)$. Throughout the time course of the stimulus, the RF center of this cell was predominantly defined by the same whisker. Like most RP cells, the immediate and far surrounds of this cell's RF changed dynamically over poststimulus time, but the RF center did not change its position in the longitudinal axis of the face. Some of the RP cells had the $\mathrm{PW}_{\mathrm{LL}}$ located in a different neighboring whisker of the same rostral column. In most of the these cases (as seen in Fig. 7), however, the $\mathrm{PW}_{\mathrm{SL}}$ belonged to the RF center defined by the LLRs $($ RM $>95 \%)$.

Finally, the six neurons not classified as $C \rightarrow R$ or $R P$ cells were observed to have either rostral-to-caudal RF center shifts $(n=2)$ or complex dynamic RFs $(n=4)$.

\section{Statistical validation of the functional classification of VPM neurons in two groups}

Overall, it appeared that three different factors contributed to the classification of VPM cells into two functionally distinct groups $\left(\mathrm{C} \rightarrow \mathrm{R}\right.$ and $\mathrm{RP}$ ). $\mathrm{C} \rightarrow \mathrm{R}$ shifting cells exhibited (1) $\mathrm{PW}_{\mathrm{SL}}$ located in the caudal whiskers, (2) larger RFs, and (3) a higher probability of displaying $\mathrm{C} \rightarrow \mathrm{R}$ shifts. $\mathrm{RP}$ cells exhibited (1) $\mathrm{PW}_{\mathrm{SL}}$ located in the more rostral whiskers, (2) smaller RFs, and (3) a much lower probability of displaying any time-dependent RF shift across the longitudinal axis of the face.

Factor analysis (FA) was used to test the statistical significance of this classification. FA is a multivariate technique commonly used to group variables according to their shared communalities. In this analysis, each cell was treated as an independent variable and their responses to all stimulated whiskers as cases. Figure $8 A$ shows a typical clustering pattern of VPM cells obtained by employing FA with a varimax rotation on data derived from 23 simultaneously recorded cells. In this scatter plot, each cell's overall pattern of response is correlated with the pattern described by the two orthogonal factors that account for most of the variance observed in the ensemble's response. Observation of the clustering pattern (ellipses) in this figure suggests that Factor 1 represents a combined measurement of overall RF size and magnitude of RF shift whereas Factor 2 represents the column position of the principal whisker at short latency.

The largest cluster (the C-R ellipse) defined by cells exhibiting a high correlation with Factor 1 and a much lower correlation with Factor 2 was entirely formed by cells previously classificd as $\mathrm{C} \rightarrow \mathrm{R}$. On the other hand, cells displaying very little correlation with Factor 1 and a somewhat larger range of correlations with Factor 2 were identified as RP neurons (divided in two "position" ellipses). Cells with complex dynamic RF (cells 3 and 10) occupied intermediary positions between the two main clusters. Cell 10 displayed a moderate $\mathrm{C} \rightarrow \mathrm{R}$ shift and cell 3 did not. Therefore, their relative locations on this plot were determined by their shifting index. Cells 7 and 24 were not clustered with these two factors.

Overall, factor analysis not only supported our RF-based classification of VPM neurons but also provided a quantitative summary of this scheme in which even rare and atypical dynamic behaviors in the sample were positioned according to their similarity to one or the other functional group. The 3-D scatter plot in Figure $8 B$ summarizes the main differences between $C \rightarrow R$ and $R P$ neurons based on the results provided by factor analysis. In this graph, as the $\mathrm{PW}_{\mathrm{SL}}$ of a single VPM cell moves from the most caudal ( 1 in the $x$-axis) to the most rostral whiskers ( 5 in the $x$-axis), neuronal RFs tend to become smaller (y-axis), and their ability to exhibit RF center shifts decreases, as measured by the shifting index (SFi, z-axis). SFi for CR cells ranged from 2 to 4 (mean $\pm \mathrm{SD}, 2.58 \pm 0.607$ ). $\mathrm{R} \rightarrow \mathrm{C}$ shift cells $(n-2)$ had negative SFi.

\section{Analysis of many-neuron ensembles: population maps}

The discovery of distinct types of spatiotemporal $\mathrm{RFs}(\mathrm{C} \rightarrow \mathrm{R}$ shifting and RP cells) suggests that VPM neuronal ensembles may be functionally heterogeneous in their responses to peripheral stimulation. Thus, their functionality appears more complex than their morphology, which has been considered to be homogeneous (Harris, 1986). Since many-neuron ensembles were recorded simultaneously in this study, it was possible to define the nature of VPM population responses to stimulation of single whiskers. To depict such population responses we have developed several 3-D and 4-D graphical procedures for representing both the spatial and temporal domains of ensemble activity during sensory responses.

Figure 9 illustrates two "population PSTHs" depicting the sensory responses of an ensemble of 23 VPM neurons to whiskers (D4 and E2) in an awake animal. These graphs show that nearly all neurons in the ensemble responded, though with different magnitudes and latencies, to the stimulation of these two whiskers. Thus, the stimulation of different single whiskers did not simply activate distinct populations of cells but instead produced different overall spatiotemporal patterns of ensemble activity. Therefore, despite the usual trial to trial variation in neuronal sensory responses, we observed that averaging re-

\footnotetext{
Figure 7. Spatiotemporal RF of a rostral position (RP) VPM cell. RP cells characteristically maintained their RF centers around the same rostral whiskers during the duration of the stimulus. Here, the RF center was defined by whisker E5. In addition, as did most of the position cells of our sample, this cell exhibited complex, time-dependent modifications in its RF surround (caudal and intermediary whiskers).
} 


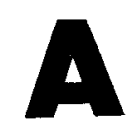

Normalized Vanmax Rotation

Normalized Factor Loadings

(computed from 4 factors of $R$ matrix)

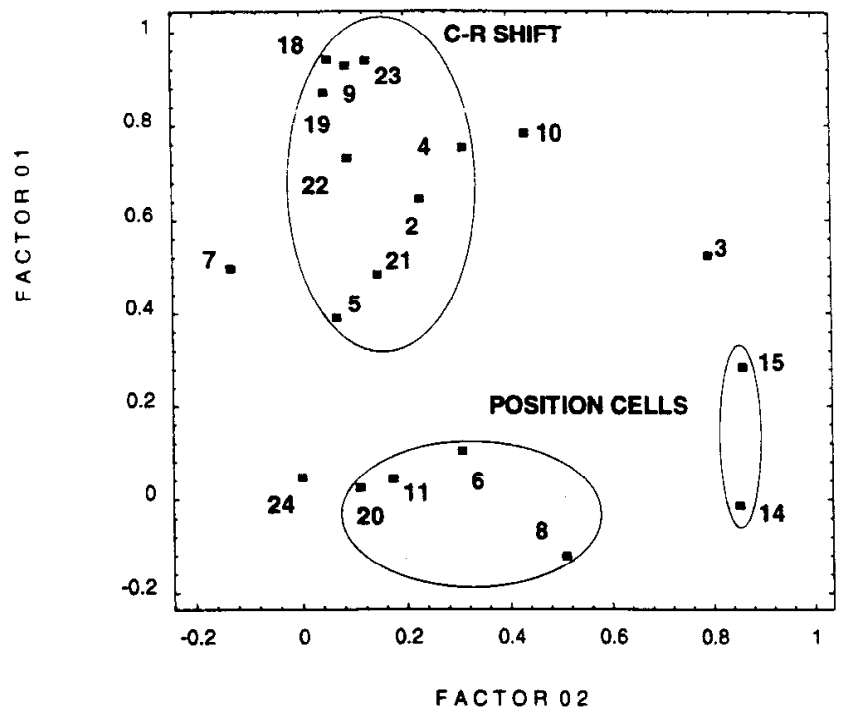

2

RF SHIFT AS A FUNCTION OF RF SIZE AND CENTER POSITION

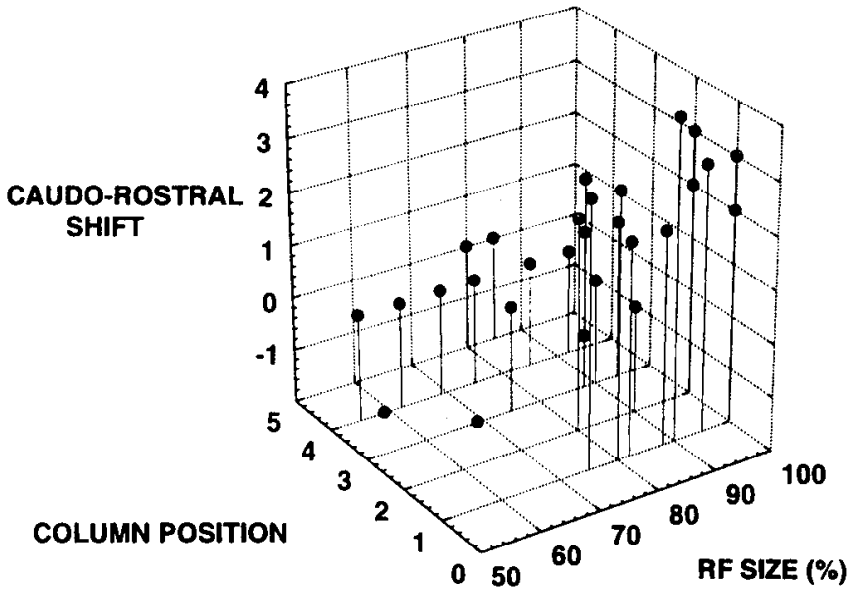

Figure 8. Functional classification of VPM neurons. A, Scatter plot depicting the correlation of the sensory responses of 23 VPM neurons, obtained by independent stimulation of 20 whiskers, to the two most significant factors derived from the application of factor analysis to the same data. Cells exhibiting high correlations with Factor 1 (vertical axes) have the largest overall RFs and the largest RF shifts. Cells highly correlated with Factor 2 had their short-latency PWs located in the most rostral whiskers while neurons with lower correlation with Factor 2 had their short-latency PWs located in progressively more caudal locations. The combination of these two factors in this scatter plot clearly differentiated two heterogeneous populations of VPM neurons: caudal-torostral $(C \rightarrow R)$ shifting cells and rostral position (RP) cells. Clusters of these cells are indicated by ellipses (one for the $\mathrm{C} \rightarrow \mathrm{R}$ shifting neurons and two for rostral position cells). $B$, A 3-D scatter plot represents the sponses to repetitive controlled whisker stimulation produced population response maps unique to each whisker. Each such map, therefore, depicts the network coding for stimulus position in that they contain differentially weighted contributions from each of the individual VPM cells in the population. This coding appears to be well distributed across the network, as evidenced by the fact that single-whisker stimulation produced some level of response in neurons recorded across multiple principal whisker domains of the VPM.

These maps also indicated patterns of functional associations between neurons that were quantitatively described using FA and principal components analysis (PCA) along with population PSTHs. Figure 10 shows the result obtained when this type of multivariate analysis was performed on data derived from an ensemble of 23 VPM cells. Stimulation of whiskers E1-E5 produced distinct population PSTHs. Scatter $x-y$ graphs in Figure 10 illustrate the degree of correlation ( -1 to 1 ) of each cell's response to the two most significant components derived from the overall sample of sensory responses. Functional associations among cells were then defined by examining the number of clusters and their membership. Stimulation of different whiskers induced variable numbers of clusters and different combinations (cluster membership) of both $\mathrm{C} \rightarrow \mathrm{R}$ and RP cells, suggesting that singlc-whisker stimulation not only induces responses throughout the VPM but also that distinct functional neuronal associations can be used to code for stimulus position.

In this context, more realistic representations of the actual functional dynamics of the rat VPM map were obtained when population spatiotemporal RFs were built. In these 4-D maps, spatial and temporal domains of sensory responses could be visualized in the context of the overall anatomical organization of the VPM. Figure $11, D$ and $E$, depicts a sequence of these population maps to illustrate how sensory information concerning the stimulation of whiskers across the $E$ row spread in space and time across an ensemble of 23 simultaneously recorded VPM neurons. This example suggests that at the ensemble level, the spatial domain of sensory responses may shift over the entire population of VPM neurons as a function of poststimulus time.

\section{Discussion}

Our development and utilization of techniques for chronic simultaneous recordings of the activity of many/single neurons have allowed a direct examination of the functional responses of VPM neuronal ensembles to independent stimulation of dif-

amount of caudal-to-rostral shift in RF center (z-axis, SFi in number of whiskers) as a function of columnar position of the short-latency $\mathrm{PW}$ ( $x$-axis) and RF size ( $y$-axis, in percentage of area tested). This graph revealed a clear linear transition from rostral position cells (caudal-torostral shift $=0$, small $R F$ size, and rostral position of short-latency PW) to $\mathrm{C} \rightarrow \mathrm{R}$ shifting neurons (caudal-to-rostral shift $>0$, large $\mathrm{RF}$ size, and short-latency $\mathrm{PW}$ in caudal whiskers). Number of points is smaller than total VPM sample because cells with similar $(\mathrm{x}, \mathrm{y}, \mathrm{z})$ values are superimposed in the graph.

Figure 9. Population PSTHs $(A$ and $B$ ) illustrate the poststimulus time course of sensory-evoked responses of a simultaneously recorded ensemble of 23 single VPM neurons in an awake rat, following the stimulation of whiskers D4 $(A)$ and E2 $(B)$. Notice that the same ensemble responds to the independent stimulation of both whiskers. Each population response, however, is characterized by different contributions from each individual cell in terms of both response magnitude and latency components. 

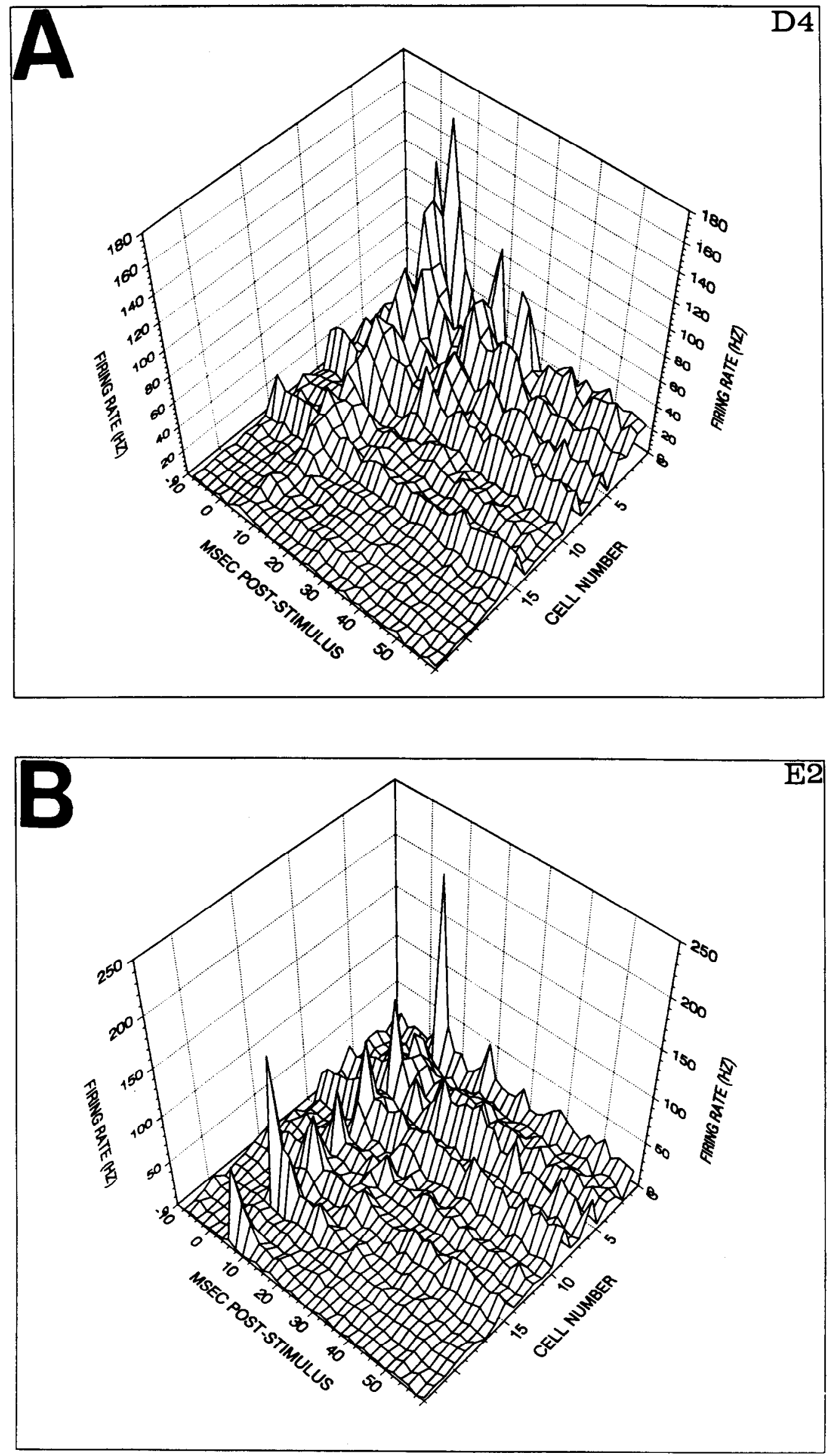

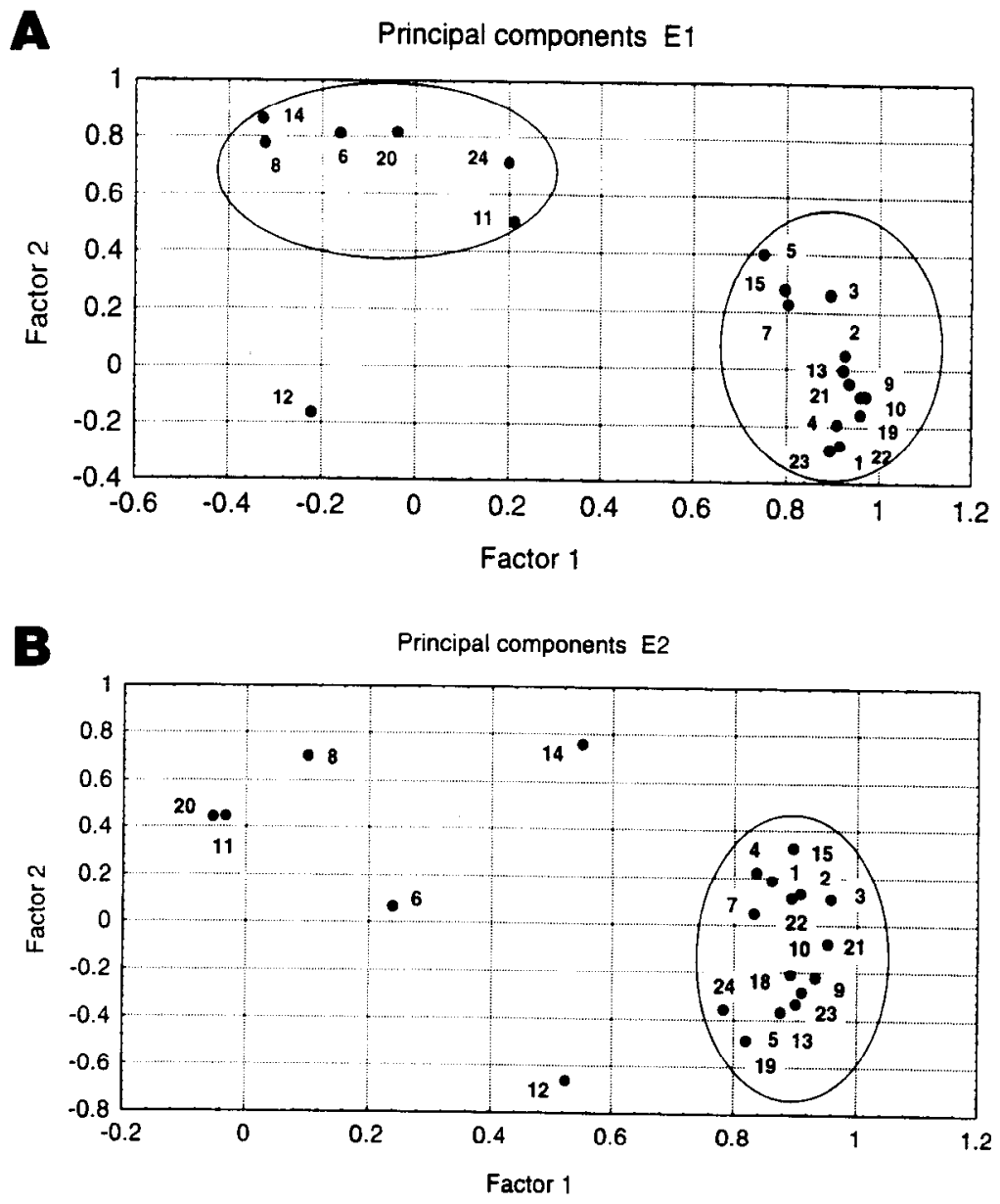

Figure 10. Use of principal components analysis to measure functional association among simultaneously recorded VPM cells. Functional associations were defined according to the correlation of each cell's sensory response with the two most significant principal components derived from the overall sensory response of an ensemble of 23 VPM neurons. Notice that repetitive stimulation of different single whiskers induced a distinct functional clustering of VPM neurons. Thus, while two main groups of cells were obtained following stimulation of whisker $\mathrm{E} 1(A)$, and only one for whisker E2 $(B)$, at least three distinct groups were obtained by stimulating whisker E5 $(C)$. The membership in each cluster also changed as a function of stimulus location.

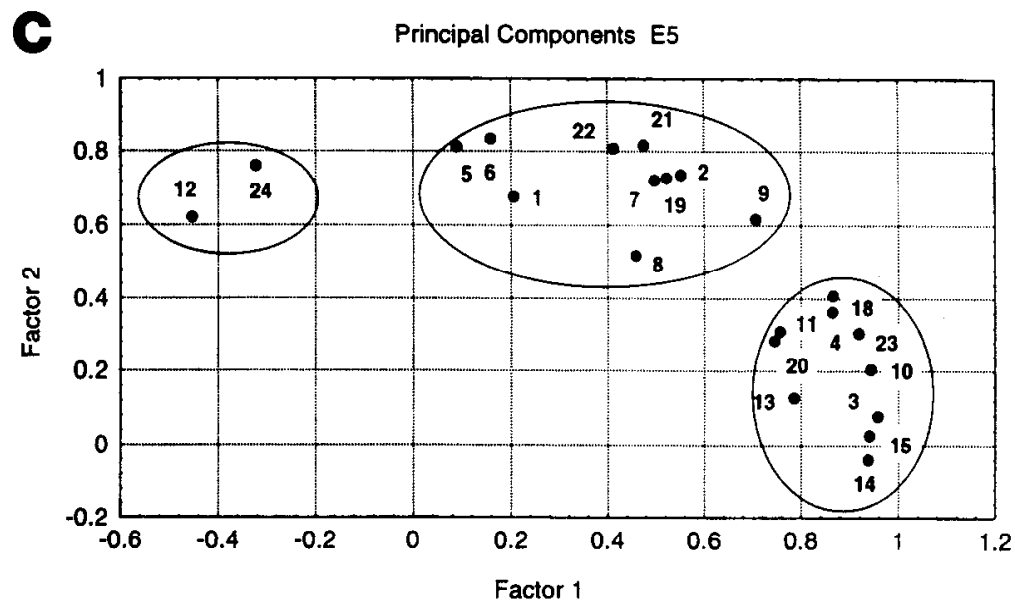

ferent facial whiskers. Quantitative analysis of these sensory responses at both the single neuron and population level revealed that the physiology of the VPM does not follow the classical single whisker $\rightarrow$ single barreloid scheme. Instead, single neuronal RFs in the VPM were found to be extremely large, with only their central peaks restricted to a single principal whisker. Though the central peaks by themselves form a sensory representation corresponding closely to the previously described topographic map in the VPM (Waite, 1973a), they seriously underrepresent the functional repcrtoire of these ncurons. In particular, such a topographic map does not account for the fact that VPM RFs may change dynamically over poststimulus time. These findings have forced us to reconsider the classical notion that single-whisker sensory information in the VPM is represented exclusively, and in a nonoverlapping fashion within single CO-rich barreloids. Furthermore, our demonstration of functionally distinct classes of neurons in the VPM (e.g., C $\rightarrow R$ vs RP neurons) suggests that the VPM is functionally heterogeneous, even though it may be morphologically homogeneous. Thus, the findings obtained here using highly quantitative neurophysiological measurements contrast with the classical view that was strongly influenced by the neuroanatomical demon- 

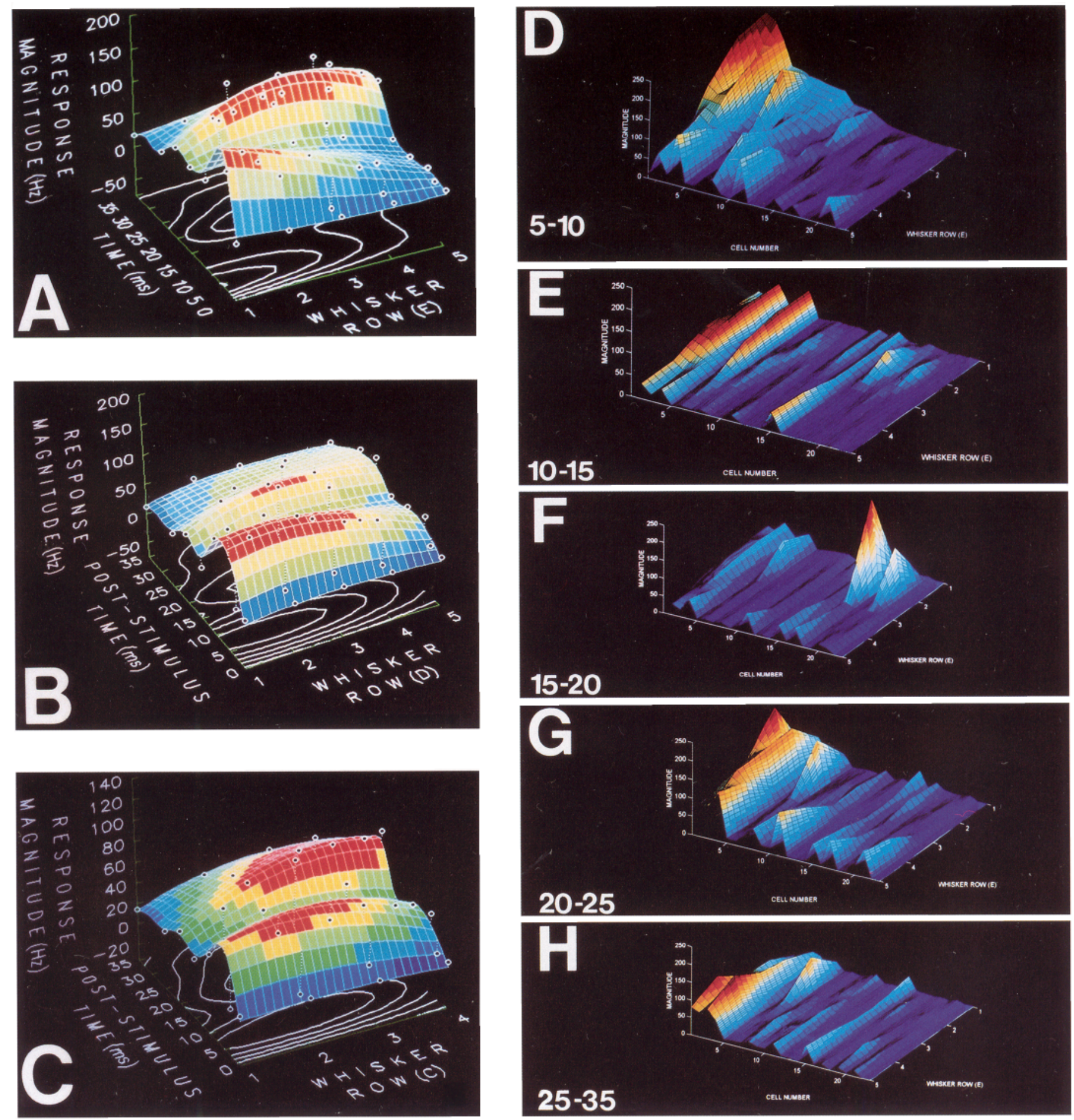

Figure 11. Single-cell $(A-C)$ and population $(D-H)$ spatiotemporal RFs depict the dynamic nature of sensory responses in the rat VPM. $A-C$, Characterization of time-dependent caudal-to-rostral RF shifts of a single VPM neuron according to different whisker rows. Notice that the magnitude of the RF shift is more prominent in whisker row $\mathrm{E}(A)$ than in rows $\mathrm{D}(B)$ or $\mathrm{C}(C) . D-H$, A sequence of five population spatiotemporal RFs depicts the time-dependent (from 5 to $35 \mathrm{msec}$ poststimulus time as indicated on the lower left corner of each graph) modifications in the spatial domain of sensory responses (RM in the $z$-axis is in $\mathrm{Hz}$ ) of an ensemble of 23 VPM neurons (1-23, x-axis) following discrete stimulation of facial whiskers (row E, $1=\mathrm{E} 1,5=\mathrm{E} 5$, y-axis) in a lightly anesthetized rat. Notice that as a result of a gradual, time-dependent population RF shift, short-latency sensory responses $(D ; 5-10 \mathrm{msec})$ that were mainly centered in the most caudal whiskers (E1 and E2) evolve to define long-latency responses $(H ; 25-35 \mathrm{msec})$ mainly located in rostral whiskers (E4 and E5). This suggests that whisker stimulation induces a spatiotemporal wave of sensory-evoked activity over most of the VPM. Open circles in $A-C$ represent actual data points. All color codes were automatically scaled according to the vertical range (red $=$ highest response, dark blue $=$ lowest) and thus vary from graph to graph. 
stration of barreloid arrays in the VPM. To reconcile these views, we here propose that the neurophysiologically defined functional representation in the VPM is much more distributed, dynamic, and heterogeneous than the anatomical representation defined by the barreloids. As is further developed in the discussion below, we believe that this definition of the functional representation provides a more rigorous and realistic substrate for information processing in this nucleus.

\section{Advantages of simultaneous many/single neuron recordings}

The ability to carry out chronic simultaneous recordings of large numbers of VPM neurons in the same animal was fundamental for the results described here. First, large-scale parallel recordings reduced dramatically the impact of nonstationary variables (such as behavioral state, level of anesthesia, degree of arousal, etc.) on data sampling. It is well known that the accurate control of the influence of nonstationary factors on classical serial singleunit recordings in anesthetized preparations is nontrivial. It requires each single cell isolated in the same animal to be recorded under the same experimental conditions (i.e., behavioral, arousal, hormonal, circadian, anesthetic, etc.), and precise reproduction of these conditions for samples obtained from different animals. As a consequence, small comparable cell samples can be obtained per animal and larger numbers of animals are necessary for building a statistically sound database. This latter factor further increases the impact of nonstationary factors on the overall data sample.

Our experimental approach solves most of these problems by allowing simultaneous recordings from the same large set of neurons per animal under identical experimental conditions. This not only increases the cell yield per animal but also reduces the overall number of animals necessary to build reasonable data samples. Furthermore, acute tissue damage is minimal and long-term damage (Fig. $1 B, C$ ) is comparable to that observed with traditional serial recordings.

Since these recordings can remain stable over long periods of time, a thorough and quantitative characterization of RFs can be carried out in the same animal under both anesthetized and awake conditions. Large-scale parallel recordings also allowed a more thorough sampling of scnsory responscs over the "map" of the face in the VPM. Heretofore, classical mapping studies, based on serial single-unit and unit-cluster recordings, have relied on large numbers of electrode penetrations to define the topographic progression of RFs across a given neuronal structure. While this approach may provide a first approximation of the functional organization of a given structure (e.g., the topographic representation of the peripheral receptor surface), it cannot represent properly the complex temporal structure of concurrent neuronal activity. Here, this "temporal domain" of sensory maps was reconstructed in 3-D plots that depict the spatiotemporal population maps embedded in ensembles of VPM cells. These population maps constituted samples of the overall wave of sensory-evoked activity that can be observed across the VPM following a peripheral stimulus. As such, they provide a much more realistic visualization of the functional dynamics of this nucleus.

\section{VPM neurons have multiwhisker $R F_{S}$}

Over many years, the epitome of the classical model of sensory processing in the rat VPM has been based upon the notion that its neuronal RFs are restricted to a single whisker. This classical view posits that "the circuitry within the VPM does little to modify the input to this nucleus from the trigeminal brain stem complex. VPM neurons appear nearly identical to PrV [principal trigeminal nucleus] cells in normal animals" (Rhoades et al., 1987).

Single-whisker RFs in the VPM were first reported by Waite (1973a), who described the topographic representation of the whisker pad within this structure by aurally monitoring the spatial distribution of sensory-evoked responses of multiunits, while whiskers and body surfaces were manually stimulated in animals anesthetized with urethane or Nembutal. Even though a majority of single-whisker RFs were found, Waite (1973a) did report that VPM RFs became larger at lighter levels of barbiturate anesthesia. Single-whisker RFs were also reported in subsequent studies (Rhoades et al., 1987; Sugitani et al., 1990; Chiaia et al., 1991b). Rhoades et al. (1987), employing the same basic recording strategy used by Waite (1973a) and relying on manual stimulation of the whiskers of animals anesthetized with xylazine and ketamine and maintained under urethane, reported that $80.3 \%$ of their VPM cell sample responded to the deflection of only a single whisker. Rhoades et al. (1987) computed the mean RF of their sample (mean $\pm \mathrm{SD}, 1.2 \pm 0.5$ whiskers) and reported that no cell responded to the stimulation of more than four whiskers. More recently, Chiaia et al. (1991b), using a similar recording strategy in deeply anesthetized animals, reported a mean VPM RF of 1.4 whiskers $(\mathrm{SD}=1.0)$ in extracellular recordings, but $2.7 \pm 1.3$ in intracellular recordings.

Despite the apparent consensus built by these studies, multiwhisker RFs in the VPM have also been reported in the literature (Semba et al., 1980). These authors suggested that multiunit activity from the VPM of awake rats defined RFs containing two to seven whiskers. More recently, this issue was readdressed by several independent studies that applied quantitative measurements to evaluate the RF size of electronically discriminated single VPM neurons, following discrete and automatically controlled stimulation of single whiskers. These produced results quite distinct from those of nonquantitative studies. Simons and Carvell (1989) reported that $71 \%$ of VPM cells exhibited multiwhisker RFs in rats anesthetized with fentanyl. The characterization of RFs in this study was limited to the stimulation of the PW and four surrounding whiskcrs. Nevertheless, a sample of 10 VPM cells analyzed yielded an average RF of 3.3 whiskers (or $66 \%$ of the area tested). Ito (1988) described a median VPM RF containing four whiskers. Armstrong-James and Callahan (1991) subsequently reported that under controlled urethane anesthesia, VPM cell RFs averaged three to five whiskers when nine vibrissae were tested. These authors suggested that increasing the anesthesia level suppressed excitatory surrounding responses and eventually led to the expression of a large proportion of single-whisker VPM RFs. Finally, Diamond et al. (1992) reported an average RF size of 4.4 whiskers in the VPM (up to 12 whiskers tested, including the PW; M. E. Diamond, personal communication) in urethane-anesthetized animals.

In the present study, "a priori" statistical criteria were used for considering sensory-evoked responses, expressed in PSTHs, as being significant $(P<0.01$ in Kolmogorov-Smirnov or ANOVA tests). These measurements were used to quantify the full extent of the RF of single VPM cells. In this context, the mean VPM RF size obtained (13.74 \pm 4.8 whiskers) was 10 times larger than the values reported in nonquantitative studies (Rhoades et al., 1987; Chiaia et al., 1991b). In fact, the mean values reported by these authors $(1.2 \pm 0.5$ and $1.4 \pm 1.0$ 
whiskers) were very similar to the one obtained in our study $(1.41 \pm 0.70$ whiskers) when the minimal response magnitude threshold used to quantify RF sizes was $95 \%$ of the $\mathrm{RM}_{\max }$ (Table 2). As such, these $\mathrm{RM}_{95 \%}$ responses closely reproduce the classical somatotopic maps that have been previously described in this area. In this sense, maps derived by metabolic techniques, such as 2-deoxyglucose (2DG), may represent only multineuronal activity above a certain response threshold, averaged over long periods of time. These tend to ignore the more subliminal responses obtained by stimulation of whiskers surrounding the PW. Here, these were found to elicit RMs that were considerably smaller than $\mathrm{RM}_{\max }$, but still highly statistically significant. Thus, our lowering of the magnitude threshold revealed increasingly larger single VPM neuron RFs (Table 2). This was made possible by employing 360 or more stimuli (in lightly anesthetized rats) for each whisker, which brought the contribution from surrounding whiskers into statistical significance. It appears, therefore, that the selection of a high-magnitude threshold, such as $\mathrm{RM}_{95 \%}$, is insufficient for measuring the overall RF size of VPM neurons.

Several important conclusions can be drawn from the above discussion. First, single-whisker VPM RFs can be obtained only in deeply anesthetized rats. Recently, an explanation for this phenomenon was proposed (M. H. Fridberg, S. M. Lee, and F. F. Ebner, unpublished observations). According to these authors, under deep levels of anesthesia one can observe a selective disruption of ascending, multiwhisker inputs from the trigeminal interpolaris subnucleus to VPM neurons. Previous studies, therefore, may have utilized deep anesthesia levels to restrict VPM neurons to single-whisker responses derived from the lessaffected principal trigeminal nucleus. These results are consistent with our contention that under more behaviorally relevant (awake) or less-artificial experimental conditions (lightly anesthetized animals), in which the overall trigeminal system is allowed to express its real functional dynamics, VPM neurons exhibit extremely large, multiwhisker fields. In fact, the dominance of these multiwhisker VPM RFs should be expected given the high degree of convergence of trigeminothalamic projections on these cells (Peschanski et al., 1983; Peschanski, 1984; Chiaia et al., 1991a).

Furthermore, our data indicate that previous assessments of RF size, based on nonquantitative measurement of sensoryevoked responses and manual stimulation of skin territories, were able to identify only the RF center of VPM neurons (their PW) but not the full extent of excitatory surrounds that together with the PW define the overall spatial domains of VPM neuronal RFs. Paradoxically, support for the existence of these multiwhisker surrounds in the VPM has been provided by Chiaia et al. (1991 b), who demonstrated that the extent of dendritic trees of VPM neurons would allow these neurons to sample information from multiple barreloids. Intracellular recordings of VPM cells carried out by the same authors also revealed the clear presence of EPSPs elicited by the stimulation of other single whiskers in addition to the PW. In a more detailed analysis, Armstrong-James and Callahan (1991) reported that the mean RM obtained by stimulating whiskers surrounding the $\mathrm{PW}$ was $25.6 \%$ of the $\mathrm{RM}_{\text {max }}$. Therefore, it is likely that traditional nonquantitative extracellular recordings lack sufficient sensitivity to detect such smaller surrounding responses. In the population context, however, the summed contribution of small surround responses can be highly significant.

Overall, our results in awake and lightly anesthetized animals rule out the hypothesis that sensory responses in the rat VPM are identical to those observed in the principal nucleus ( $P r V)$ of the brainstem trigeminal complex. This conclusion has been recently reinforced using the same recording and analysis technique described here to characterize RFs in the PrV. These experiments (Nicolelis et al., 1993c) demonstrated that PrV cells have much smaller RFs than VPM neurons and almost never exhibit LLRs in response to the same whisker stimulation parameters used in the present study. Thus, the proposition of a local-coding scheme, based on single-whisker RFs throughout the rat lemniscal trigeminal pathway (Rhoades et al., 1987; Killackey et al., 1990; Chiaia et al., 1991b), can no longer be supported, given the existence of multiwhisker RFs in the rat VPM, the primary thalamic relay of this pathway.

\section{Spatiotemporal nature of thalamic RFS}

A further level of complexity of RFs in the VPM was provided by the existence of both short- and long-latency responses. The latency range (15-35 msec) of LLRs matched similar observations obtained with punctate stimulation ( $3 \mathrm{msec}$ duration; see Armstrong-James and Callahan, 1991). This confirms our unpublished observations using short-duration stimuli and suggests that the presence of LLRs was not dependent on the sustained (100 msec) whisker displacement used here.

Although LLRs have been observed previously in the VP thalamus (Shin and Chapin, 1990; Armstrong-James and Callahan, 1991), no systematic analysis of their influences on neuronal RFs has preceded the present study. Surprisingly, the overall RFs defined by the LLRs were smaller than the ones defined by SLRs, at least in the $\mathrm{C} \rightarrow \mathrm{R}$ class of VPM neurons. In these same neurons, the RF centers (the $\mathrm{PW}_{\mathrm{LL}}$ ) defined by these LLRs were remarkably different from those defined by the SLRs. The probability of exhibiting these RF shifts was correlated with different populations of VPM neurons. Thus, while virtually all cells with $\mathrm{PW}_{\mathrm{SL}}$ located in the two most caudal columns of whiskers and the straddle whiskers exhibited time-dependent RF center shifts, such shifts were rarely found in cells with $P W_{S L}$ centered in more rostral columns. In addition, time-dependent shifts were clearly biased toward caudal-to-rostral movements of the RF center over poststimulus time.

The existence of such continuous RF center shifts and timedependent changes in excitatory surrounds suggests that, contrary to the traditional model, single neuronal RFs in the rat VPM are in fact dynamic representations of the whisker pad containing both spatial and temporal domains. In this context, RFs are better represented by surfaces that represent the probability of firing of a given cell as a function of both stimulus location and poststimulus time.

\section{Possible circuit mechanisms for spatiotemporal dynamic properties}

Inspection of 3-D and 4-D representations of dynamic spatiotemporal RFs in the VPM suggests that they emerge as a consequence of temporally asynchronous convergence to this nucleus of multiple excitatory and inhibitory afferents, each of which is characterized by different RF sizes and center location. In this model, SLRs (up to $10 \mathrm{msec}$ ) might result from the convergence on VPM neurons of fast ascending somatosensory inputs not only from the PrV (Armstrong-James and Callahan, 1991) but also from the spinal trigeminal complex. In support of this, we have recently found that $\mathrm{SpV}$ neurons respond at 
short latency to low-threshold whisker stimuli, but have much larger RFs than PrV neurons (Nicolelis et al., 1993c).

Since the rat VPM lacks inhibitory interneurons, the postexcitatory inhibition observed in the responses of these neurons was most likely derived from the reticular $(\mathrm{Rt})$ nucleus of the thalamus. Most Rt neurons have large cutaneous RFs and exhibit long-lasting, reverberating oscillatory responses following stimulation of single whiskers (Fisher et al., 1992). Even though the precise spatial distribution of GABAergic Rt projections on VPM neurons is not known, it is conceivable that the density and degree of convergence of these inhibitory projections may be important for sculpting the excitatory LLRs in these neurons.

A precise definition of the source of LLRs in the VPM, however, proved more difficult. While it is very plausible to assume that slow-conducting ascending trigeminothalamic pathways could contribute somewhat to these LLRs, no experimental evidence favoring this hypothesis has been published so far. Thus, even though one cannot rule out the contribution of trigeminothalamic pathways, the latency range of these secondary VPM responses (15-25 $\mathrm{msec})$ suggests that they could very well derive from excitatory corticothalamic afferents that may be able to elicit responses on VPM cells $11 \mathrm{msec}$ after the stimulus delivery (Armstrong-James and Callahan, 1991). Intracellular recordings in brain slices have revealed that activation of corticothalamic afferents can produce prolonged excitatory postsynaptic potentials (EPSPs) in the guinea pig dorsal lateral geniculate nucleus of the thalamus (McCormick and Krosigk, 1992). This further supports our proposition that sensory information derived from corticothalamic projections could be incorporated in the process of somatosensory information representation in the thalamus.

This hypothesis, that the SLRs and LLRs have independent sources, is supported by several additional observations from our laboratory: LLRs define much smaller RFs, at any RM threshold, than SLRs in the same $C \rightarrow R$ shifting cells. RF sizes defined by LLRs in shifting cells are identical to those defined by LLRs of RP cells. Subcutaneous facial injections of lidocaine that block the SLRs of VPM cells often do not affect, or even may enhance, LLRs of the same cclls (Nicolclis ct al., 1993b). Finally, preliminary evidence indicates that LLRs can be modulated or even eliminated in favor of SLRs when focal lesions are produced in the SI cortex.

\section{Functional heterogeneity in the rat VPM: a possible behavior correlate}

The quantitative analysis of RF properties, based exclusively on low-threshold stimulation of facial whiskers, demonstrated that at least two statistically distinct functional groups can be identified in the VPM $(\mathrm{C} \rightarrow \mathrm{R}$ shifting cells and RP cells). The statistical significance of this general functional classification of VPM neurons was demonstrated by the fact that the same basic scheme is obtained when factor analysis is performed on the raw PSTH data describing the responses of simultaneously recorded neurons to all the single whiskers stimulated, without further data manipulation.

Interestingly, the functional clustering of VPM cells in two groups proposed here matches the scheme suggested by Carvell and Simons (1990) to classify different groups of facial whiskers according to their contribution for the whisking behavior exhibited by rats during tactile discrimination tasks. Rats use their more caudal whiskers, which are longer and more mobile, to swcep a surface back and forth at a frequency around $8 \mathrm{IIz}$. On the other hand, the more rostral whiskers, which are shorter and much less mobile, may remain in contact with the target surface for longer time periods. Carvell and Simons (1990) suggested that this latter group may be specialized to detect the position of objects while more caudal whiskers would be mainly involved with fine tactile discrimination of surface texture. In this context, it is tempting to speculate that the peculiar dynamic behavior of VPM neurons in rats may reflect and/or is determined by their association with a particular functional channel of the somatosensory system. In this line of reasoning, the ability to exhibit $\mathrm{C} \rightarrow \mathrm{R}$ shifts would be associated with neurons that belong to the pathway for fine tactile discrimination. Likewise, the lack of such dynamic ability would characterize neurons specialized to code for object position.

\section{Functional versus anatomic maps}

As a whole, the "functional map" embedded in VPM ensembles differed markedly from the classical topographic "anatomical" CO-based map observed in this nucleus. While the discrete and static "anatomical" map reproduces the distribution of facial whiskers in the rat snout, VPM "functional maps" may constitute thalamic images of the dynamic solutions reached by the overall trigeminal network to represent and process spatiotemporal interactions between cutaneous stimuli and the peripheral receptor surface. Therefore, even though the CO-based anatomical map may impose circuitry-related constraints on the expression of functional representations, anatomical and functional maps are not perfectly superimposed in the rat VPM because, in fact, they represent different entities. In essence, our observations lend support to the argument that prominent iterated modular units, such as the CO-rich patches in the VPM, provide a rather incomplete image of the functional dynamic properties of neuronal ensembles (Purves et al., 1992).

The functional properties of VPM ensembles described in this study are reminiscent of the type of dynamic behavior observed in hidden unit layers of artificial neural networks based upon highly distributed representations of sensory information (Churchland and Sejnowski, 1992). In a distributed scheme of coding, sensory stimuli are represented by an activation vector (i.e., a "functional representation") that is formed by the weighted contribution of many cells. In this scenario, single elements (neurons) have coarsely coded, and overlapping RFs and different sensory stimuli generate distinct activation vectors. Accordingly, the distinct VPM population responses and peculiar functional clustering of neurons, observed after the stimulation of different facial whiskers, define multiple VPM activation vectors.

Contrary to what may be intuitively expected, coarsely coded neural networks are capable of expressing extremely precise sensory discrimination ability (Churchland and Sejnowski, 1992). Therefore, instead of degrading the ability of the trigeminal system to attain fine tactile discrimination, distributed processing in the VPM may decisively contribute to the completion of this task.

Such distributed population coding has becn identified in recent years as a major functional strategy used by ensembles of cortical motor neurons in primates to code for movement direction (Georgopoulos et al., 1986, 1993). A sparse population code has also been recently proposed to explain how ensembles of neurons in the inferotemporal cortex represent images such as faces (Young and Yamane, 1992). The representation of large saccadic gaze shifts in the superior colliculus of head-free cats also seems to be obtained through a dynamic and distributed 
motor map (Guitton, 1993). As far as we know, our results are the first to suggest that a primary sensory thalamic relay nucleus may also process information through a distributed population code. This may indicate that population coding is a common neuronal strategy used in sensorimotor neural networks and in a wide range of species.

\section{Are dynamic and heterogeneous spatiotemporal RFs peculiar to the rat VPM?}

The existence of dynamic spatiotemporal thalamic RFs, which exhibit distinct excitatory or inhibitory spatial domains over poststimulus time, has been previously reported by Stevens and Gerstein (1976) in their studies of the cat LGN. These authors demonstrated that the classical definition of "on," "off," "center," and "surround" was insufficient to describe even the simplest LGN RFs. In their sample, they observed cells that displayed spatially homogeneous distributions of excitatory and inhibitory domains but also cells that exhibited spatially heterogeneous distributions of domains. They further suggested that heterogeneous RFs corresponded to X cells and homogeneous RFs corresponded to Y cells. Therefore, they not only demonstrated that "time is at least as important an analytical variable as space to visual neurons" but also associated different dynamic behaviors (homogeneous and heterogeneous) to distinct functional channels in the visual thalamus (Stevens and Gerstein, 1976).

Dinse et al. (1991) have also demonstrated that visual cortical neurons in area 17 of cats have spatiotemporal RFs that can exhibit either shifts in RF center or change in RF size as a function of poststimulus time. In fact, these authors identified two main classes of neurons based on their RF dynamics. Type I neurons exhibited little change in RF size over time but displayed important RF center shift. Type II cells exhibited no RF center shift but their RF sizes changed as a function of time (Dinse et al., 1991).

The similarity of RF classes observed here and in these two other studies is striking. As in Dinse et al. (1991), we also observed two main classes of neurons ( $\mathrm{C} \rightarrow \mathrm{R}$ shifting and $\mathrm{RP}$ cells) that seem to correspond to the heterogeneous and homogeneous classes of LGN neurons described by Stevens and Gerstein (1976). These similarities suggest that dynamic changes in the spatial domain of RFs can be observed in both cortical and thalamic neurons in different species. More recently, the properties and postnatal development of spatiotemporal RFs of simple cells in the cat's striate cortex have been characterized (DeAngelis et al., 1993a,b). Interestingly, these authors report that spatial properties of RFs mature much earlier than their temporal properties, suggesting that the dynamic attributes, which define functional maps, require more developmental experience to be established. Altogether these studies indicate that the classical notion of RFs must be reformulated to incorporate the growing experimental evidence that sensory response properties change as a function of poststimulus time and also context (Allman et al., 1985).

\section{Dynamic and distributed processing as a substrate for sensory plasticity}

The existence of substantial sensory plasticity following peripheral deafferentation in adult animals has been demonstrated repeatedly in the last 10 years (Kaas et al., 1983; Kaas, 1991). Initial results suggested that deafferentation-induced sensory reorganization would require a long period of time to be estab- lished. Nevertheless, immediate sensory reorganization of cortical (Calford and Tweedale, 1988, 1991; Gilbert and Wiesel, 1992) and thalamic (Nicolelis et al., 1993b) RFs has been recently demonstrated. In fact, simple occlusion of the RF of cortical cells, paralleled by stimulation of surrounding areas, is enough to induce an almost immediate, fivefold expansion in these RFs (Pettet and Gilbert, 1992). Altogether, experimental cvidence suggests that cortical and thalamic RFs in both visual and somatosensory pathways may be defined by an active and dynamic equilibrium of convergent afferents. The relative synaptic weight of each of these contributors may vary according to the spatiotemporal character of the stimulus. As such, sensory responses that are normally reduced or even masked could be expressed through an alteration in the balance of synaptic strengths of excitatory and inhibitory inputs to a given population of cells. Thus, the existence of coarsely coded and dynamic RFs in sensory pathways provides a suitable initial framework to explain both the occurrence of immediate sensory plasticity and the induction of changes in RF obtained during basic forms of learning such as Pavlovian conditioning (Weinberger et al., 1993). It remains to be investigated, however, whether these immediate changes occurring at both cortical and subcortical levels are fundamental to determine the course of long-term sensory plastic reorganization.

\section{References}

Abeles M, Goldstein M (1977) Multispike train analysis. IEEE Proc 65:762-773.

Allman J, Miezin F, McGuinness E (1985) Stimulus specific responses from beyond the classical receptive field: neurophysiological mechanisms for local-global comparisons in visual neurons. Annu Rev Neurosci 8:407-430.

Armstrong-James M, Callahan CA (1991) Thalamo-cortical processing of vibrissal information in the rat. II. Spatiotemporal convergence in the thalamic ventroposterior medial nucleus (VPm) and its relevance to generation of receptive fields of $\mathrm{S} 1$ cortical "barrel" neurones. J Comp Neurol 303:211-224.

Arvidson J (1982) Somatotopic organization of vibrissae afferents in the trigeminal sensory nuclei of the rat studies by transganglionic transport of HRP. II Comp Neurol 211:84-92.

Belford GR, Killackey HP (1979a) Vibrissae representation in subcortical trigeminal centers of the neonatal rat. J Comp Neurol 183: 305-322.

Belford GR, Killackey HP (1979b) The development of vibrissae representation in subcortical trigeminal centers of the neonatal rat. $J$ Comp Neurol 188:63-74.

Benson DL, Isackson PJ, Gall CM, Jones EG (1992) Contrasting patterns in the localization of glutamic acid decarboxylase and $\mathrm{Ca}^{2+}$ calmodulin protein kinase gene expression in the rat central nervous system. Neuroscience 46:825-849.

Calford MB, Tweedale R (1988) Immediate and chronic changes in responses of somatosensory cortex in adult flying-fox after digit amputation. Nature 332:446-448.

Calford MB, Tweedale $\mathrm{R}$ (1991) Immediate expansion of receptive fields of neurons in area $3 \mathrm{~b}$ of macaque monkeys after digit denervation. Somatosens Mot Res 8:249-260.

Chiaia NL, Rhoades RW, Bennett-Clarke CA, Fish SE, Killackey HP (1991a) Thalamic processing of vibrissal information in the rat. I. Afferent input to the medial ventral posterior and posterior nuclei. $J$ Comp Ncurol 314:201-216.

Chiaia NL, Rhoades RW, Fish SE, Killackey HP (1991b) Thalamic processing of vibrissal information in the rat: II. Morphological and functional properties of medial ventral posterior nucleus and posterior nucleus neurons. J Comp Neurol 314:217-236.

Chmielowska J, Carvell GE, Simons DJ (1989) Spatial organization of thalamocortical and corticothalamic projection systems in the rat SmI barrel cortex. J Comp Neurol 285:325-338.

Churchland PS, Sejnowski TJ (1992) The computational brain. Cambridge, MA: MIT Press.

DeAngelis GC, Ohzawa I, Freeman RD (1993a) Spatiotemporal or- 
ganization of simple-cell receptive fields in the cat's striate cortex. I General characteristics and postnatal development. J Neurophysiol 69:1091-1117.

DeAngelis GC, Ohzawa I, Freeman RD (1993b) Spatiotemporal organization of simple-cell receptive fields in the cat's striate cortex. II. Linearity of temporal and spatial summation. J Neurophysiol 69 : 1118-1135.

Diamond ME, Armstrong-James M, Ebner FF (1992) Somatic sensory responses in the rostral sector of the posterior group (POm) and in the ventral posterior medial nucleus (VPM) of the rat thalamus. J Comp Neurol 318:462-476.

Dinse HR, Kruger K, Mallot HA, Best J (1991) Temporal structure of cortical information processing: cortical architecture, oscillations, and non-separability of spatio-temporal receptive field organization In: Neuronal cooperativity (Kruger J, ed), pp 68-104. Berlin: Springer.

Dorfl J (1982) The musculature of the mystacial vibrissae of the white mouse. J Anat 135:147-154.

Fisher TM, Nicolelis MAL, Chapin JK (1992) Sensory and oscillatory properties of simultaneously recorded multi-single units in the thalamic reticular nucleus of the rat. Soc Neurosci Abstr 18:1392.

Gcorgopoulos AP, Swartz AB, Ketter RE (1986) Neuronal population coding of movement direction. Science 233:1416-1419.

Georgopoulos AP, Taira M, Lukashin A (1993) Cognitive neurophysiology of the motor cortex. Science 260:47-52.

Gilbert CD, Wiesel TN (1992) Receptive field dynamics in adult primary visual cortex. Nature 356:150-152.

Guitton D (1993) Control of eye-head coordination during orienting gaze shifts. Trends Neurosci 15:174-179.

Harris RM (1986) Morphology of physiologically identified thalamocortical relay neurons in the rat ventrobasal thalamus. J Comp Neurol 251:491-505.

Ito M (1988) Response properties and topography of vibrissa-sensitive VPM neurons in the rat. J Neurophysiol 60:1181-1197.

Johnson LW, Ries RD (1982) Numerical analysis. Reading: Addison Wesley.

Johnson RA, Wichern DW (1988) Applied multivariate statistical analysis. Englewood Cliffs, NJ: Prentice-Hall.

Kaas JH (1991) Plasticity of sensory and motor maps in adult mammals. Annu Rev Neurosci 14:137-167.

Kaas JH, Merzenich MM, Killackey HP (1983) The organization of the somatosensory cortex following peripheral nerve damage in adult and developing mammals. Annu Rev Neurosci 6:325-356.

Killackey HP, Jacquin MF, Rhoades RW (1990) Development of somatosensory system structures. In: Development of sensory systems in mammals (Coleman JR, ed), pp 403-428. New York: Wiley.

I and PW, Simons DJ (1985) Metabolic and structural correlates of the vibrissae representation in the thalamus of the adult rat. Neurosci Lett 60:319-324.

McCormick DA, Krosigk Mv (1992) Corticothalamic activation modulates thalamic firing through glutamate "metabotropic" receptors. Proc Natl Acad Sci USA 89:2774-2778.

Minderhoud JM (1971) An anatomical study of the efferent connections of the thalamic reticular nucleus. Exp Brain Res 12:435-446.

Nicolelis MAL, Lin RCS, Woodward DJ, Chapin JK (1993a) Dynamic and distributed properties of many-neuron ensembles in the ventral posterior medial thalamus of awake rats. Proc Natl Acad Sci USA 90:2212-2216.

Nicolelis MAL, Lin RCS, Woodward DJ, Chapin JK (1993b) Induction of immediate spatiotemporal changes in thalamic networks by peripheral block of ascending cutaneous information. Nature 361 : 533-536.

Nicolelis MAL, Lin RCS, Chapin JK (1993c) Chronic, simultaneous recordings of ensembles of single neurons across all levels of the trigeminal pathway in awake rats. Observing the functional dynamics of a sensory pathway at work. Soc Neurosci Abstr 19:105.

Paxinos G, Watson C (1986) The rat brain in stereotaxic coordinates. San Diego: Academic.

Peschanski M (1984) Trigeminal afferents to the diencephalon in the rat. Neuroscience 12:465-487.

Peschanski M, Mantyh PM, Besson JM (1983) Spinal afferents to the ventrobasal thalamic complex in the rat: an anatomical study using wheat-germ agglutinin conjugated to horseradish peroxidase. Brain Res 278:240-244.

Pettet MW, Gilbert CD (1992) Dynamic changes in receptive-field size in cat primary visual cortex. Proc Natl Acad Sci USA 89:83668370

Purves D, Riddle DR, LaMantia A-S (1992) Iterated patterns of brain circuitry (or how the cortex gets its spots). Trends Neurosci 15:362368.

Rausell E, Jones EG (199 la) Histochemical and immunocytochemical compartments of the thalamic VPM nucleus in monkeys and their relationship to the representational map. J Neurosci 11:210-225.

Rausell E, Jones EG (1991b) Chemically distinct compartments of the thalamic VPM nucleus in monkeys relay principal and spinal trigeminal pathways to different layers of the somatosensory cortex. J Neurosci 11:226-237.

Rhoades RW, Belford GR, Killackey HP (1987) Receptive-field properties of rat ventral posterior medial neurons before and after selective kainic acid lesions of the trigeminal brain stem complex. J Neurophysiol 57:1577-1600.

Seber GAF (1984) Multivariate observations. New York: Wiley.

Semba K, Szechtman H, Komisaruk BR (1980) Synchrony among rhythmical facial tremor, neocortical "alpha" waves, and thalamic non-sensory neuronal bursts in intact awake rats. Brain Res 195:281298.

Shin HC, Chapin JK (1990) Mapping the effects of motor cortex stimulation on somatosensory relay neurons in the rat thalamus: direct responscs and afferent modulation. Brain Res Bull 24:257-265.

Simons DJ, Carvell GE (1989) Thalamocortical response transformation in the rat vibrissa/barrel system. J Neurophysiol 61:311-330.

Stevens JK, Gerstein GL (1976) Spatiotemporal organization of cat lateral geniculate receptive fields. J Neurophysiol 39:213-238.

Sugitani M, Yano J, Sugai T, Ooyama H (1990) Somatotopic organization and columnar structure of vibrissae representation in the rat ventrobasal complex. Exp Brain Res 81:462-474.

Van der Loos H (1976) Barreloids in mouse somatosensory thalamus. Neurosci Lett 2:1-6.

Waite PME (1973a) Somatotopic organization of vibrissal responses in the ventro-basal complex of the rat thalamus. J Physiol (Lond) 228:527-540.

Waite PME (1973b) The responses of cells in the rat thalamus to mechanical movements of the whiskcrs. J Physiol (Lond) 228:541561 .

Weinberger NM, Javid R, Lepan B (1993) Long-term retention of learning-induced receptive-field plasticity in the auditory cortex. Proc Natl Acad Sci USA 90:2394-2398.

Williams MN, Zahm DS, Jacquin MF (1991) Differential synaptic organization of the trigeminal principalis and interpolaris projections to thalamic barreloids. Soc Neurosci Abstr 17:622.

Woolsey TA, Van der Loos H (1970) The structural organization of layer IV in the somatosensory region (SI) of mouse cerebral cortex: the description of a cortical field composed of discrete cytoarchitectonic units. Brain Res 17:205-242.

Young MP, Yamane S (1992) Sparse population coding of faces in the inferotemporal cortex. Science 256:1327-1331. 